\title{
A UNIFYING THEORY OF A POSTERIORI ERROR CONTROL FOR NONCONFORMING FINITE ELEMENT METHODS
}

\author{
C. CARSTENSEN` AND JUN HU
}

\begin{abstract}
Residual-based a posteriori error estimates were derived within one unifying framework for lowest-order conforming, nonconforming, and mixed finite element schemes in [C. Carstensen, Numerische Mathematik 100 (2005) 617-637]. Therein, the key assumption is that the conforming first-order finite element space $V_{h}^{c}$ annulates the linear and bounded residual $\ell$ written $V_{h}^{c} \subseteq$ ker $\ell$. That excludes particular nonconforming finite element methods (NCFEMs) on parallelograms in that $V_{h}^{c} \not \subset$ ker $\ell$. The present paper generalises the aforementioned theory to more general situations to deduce new a posteriori error estimates, also for mortar and discontinuous Galerkin methods. The key assumption is the existence of some bounded linear operator $\Pi: V_{h}^{c} \rightarrow V_{h}^{n c}$ with some elementary properties. It is conjectured that the more general hypothesis (H1)-(H3) can be established for all known NCFEMs. Applications on various nonstandard finite element schemes for the Laplace, Stokes, and Navier-Lamé equations illustrate the presented unifying theory of a posteriori error control for nonconforming finite element methods.
\end{abstract}

\section{Unified Mixed Approach to Error Control}

Suppose that the primal variable $u \in V$ (e.g., the displacement field) is accompanied by a dual variable $p \in L$ (e.g., the flux or stress field). Typically $L$ is some Lebesgue and $V$ is some Sobolev space; suppose throughout this paper that $L$ and $V$ are Hilbert spaces and $X:=L \times V$. Given bounded bilinear forms

$$
a: L \times L \rightarrow \mathbb{R} \quad \text { and } \quad b: L \times V \rightarrow \mathbb{R}
$$

and well established conditions on $a$ and $b[16,20]$, the linear and bounded operator $A: X \rightarrow X^{*}$, defined by

$$
(A(p, u))(q, v):=a(p, q)+b(p, v)+b(q, u),
$$

is bijective. Then, given right-hand sides $f \in L^{*}$ and $g \in V^{*}$, there exists some unique $(p, u) \in X$ with

$$
\begin{aligned}
a(p, q)+b(q, u) & =f(q) & & \text { for all } q \in L \\
b(p, v) & =g(v) & & \text { for all } v \in V .
\end{aligned}
$$

Date: October 31, 2006.

2000 Mathematics Subject Classification. 65N10, 65N15, 35J25.

Key words and phrases. a posteriori error analysis, finite element method, nonconforming finite element method, error estimates reliability.

The first author CC was supported by DFG Research Center MATHEON "Mathematics for key technologies" in Berlin and the German Indian Project DST-DAAD (PPP-05). The second author JH was partially supported by National Science Foundation of China under Grant No.10601003. 
Suppose $\left(p_{h}, \tilde{u}_{h}\right) \in L \times V$ is some approximation to $(p, u)$ and define

$$
\begin{aligned}
& \mathcal{R e s}_{L}(q):=f(q)-a\left(p_{h}, q\right)-b\left(q, \tilde{u}_{h}\right) \quad \text { for all } q \in L, \\
& \mathcal{R e s}_{V}(v):=g(v)-b\left(p_{h}, v\right) \text { for all } v \in V .
\end{aligned}
$$

Here and throughout, $\tilde{u}_{h}$ is some continuous and not necessarily discrete function established as the key ingredient in [23]; however, the subindex in $\tilde{u}_{h}$ refers to the fact that $\tilde{u}_{h}$ might be closely related (or designed with some post-processing) to some discrete function $u_{h}$ and hence that $\tilde{u}_{h}$ is on our disposal. Since $A: X \rightarrow X^{*}$ is an isomorphism, there holds

$$
\left\|p-p_{h}\right\|_{L}+\left\|u-\tilde{u}_{h}\right\|_{V} \approx\left\|\mathcal{R} e s_{L}\right\|_{L^{*}}+\left\|\mathcal{R} e s_{V}\right\|_{V^{*}}
$$

Here and throughout, an inequality $a \lesssim b$ replaces $a \leq C b$ with some multiplicative mesh-size independent constant $C>0$ that depends only on the domain $\Omega$ and the shape (e.g., through the aspect ratio) of elements $(C>0$ is also independent of crucial parameters as the Lamè parameter $\lambda$ below). Finally, $a \approx b$ abbreviates $a \lesssim b \lesssim a$.

Remark 1.1. Note that (1.3)-(1.4) are a primal mixed formulation with $L:=L^{2}(\Omega)^{m \times n}$ for the Laplace, Stokes, and Navier-Lamé equations under consideration. Throughout this paper, the discrete component $p_{h}$ is derived from $u_{h}$, e.g, $p_{h}=\nabla_{\mathcal{T}} u_{h}$ in case $p=\nabla u$ for the Laplace equation; while $u_{h}$ is solved from the discrete problem in the displacement-oriented formulation (Sections 4-6 below).

The examples in [23] include conforming, nonconforming and mixed finite element schemes for the Laplace, Stokes, and Navier-Lamé equations. This paper will consider such applications in Section 4, 5, and 6 below for with focus on NCFEMs displayed in Table 4.1, 5.1, 6.1, and 6.2. The applications of the present theory to mortar and discontinuous Galerkin methods are also condidered in Section 4 for the Poisson problem. Therein, the norms of $\mathcal{R} e s_{L}$ and $\mathcal{R} e s_{V}$ are estimated under the general hypothesis that each of those has the form

$$
\mathcal{R} e s(v):=\int_{\Omega} g \cdot v d x+\int_{\cup \mathcal{E}} g_{\mathcal{E}} \cdot v d s \quad \text { for } v \in V .
$$

Here and below, $V$ belongs to some Sobolev space $V=H_{0}^{1}(\Omega)^{m}$ and $g \in L^{2}(\Omega)^{m}$, while $g_{\mathcal{E}} \in L^{2}(\cup \mathcal{E})^{m}$ with some domain $\Omega \subset \mathbb{R}^{n}$ and the union $\cup \mathcal{E}$ of edges (if $n=2$ ) or faces (if $n=3$ ) related to a regular triangulation of $\Omega$. Some required key property in [23] on both $\mathcal{R} e s=\mathcal{R} e s_{L}$ and $\mathcal{R} e s=\mathcal{R} e s_{V}$ reads

$$
V_{h}^{c} \subset \operatorname{ker} \mathcal{R} e s \subset V \text {. }
$$

In this situation, a typical result of an explicit residual-based error estimation reads

$$
\|\mathcal{R} e s\|_{V^{*}}^{2} \lesssim\left\|h_{\mathcal{T}} g\right\|_{L^{2}(\Omega)}^{2}+\sum_{E \in \mathcal{E}} h_{E}\left\|g_{\mathcal{E}}\right\|_{L^{2}(E)}^{2}=: \eta^{2} .
$$

Here and throughout, $h_{\mathcal{T}}$ and $h_{E}$ denote local mesh-sizes in the underlying triangulation, i.e.,

$$
\left.h_{\mathcal{T}}\right|_{T}=\operatorname{diam}(T) \text { for any } T \in \mathcal{T} \text {, and } h_{E}=\operatorname{diam}(E) \text { for any } E \in \mathcal{E} .
$$


$V_{h}^{c}$ includes the first-order finite element functions to ensure (1.10). Details on the notation and the concrete examples will be given below. The terms in (1.8) often result from some discretisation of the equilibration condition (1.4),e.g., via an integration by parts, and hence the term $\mathcal{R} e s_{V}$ is referred to as the equilibration residual.

The first aim of this paper is the generalisation of (1.10) for $\mathcal{R} e s=\mathcal{R}_{e} s_{V}$ in Theorem 2.1 of Section 2 to allow the control of certain nonstandard finite element schemes without the condition (1.9) in Sections 4-6. Here, one key theory is to replace (1.9) by assumptions (H1)-(H3) on some Clement-type operator $J$ and some linear bounded operator $\Pi$ between the conforming and nonconforming finite element spaces.

For the Laplace, Stokes, and Navier-Lamé equations considered herein, one can observe from the definitions of $a(\cdot, \cdot)$ and $b(\cdot, \cdot)$ in Sections $4-6$ below that the consistency residuum $\mathcal{R} e s_{L}$ from (1.5) can also be written in the form (1.8). With some bounded linear operator $\mathcal{A}: L:=L^{2}(\Omega)^{m \times n} \rightarrow L$, the norm of $\mathcal{R} e s_{L}$ allows the form

$$
\min _{\tilde{u}_{h} \in V}\left\|\mathcal{R} e s_{L}\right\|_{L^{*}} \approx \min _{\tilde{u}_{h} \in V}\left\|\mathcal{A}\left(p_{h}\right)-D \tilde{u}_{h}\right\|_{L^{2}(\Omega)} .
$$

Therein $D \tilde{u}_{h}$ denotes the functional matrix of all first-order partial derivatives (e.g., the gradient and possibly also the Green strain of linear elasticity) of the Sobolev function $\tilde{u}_{h}$ in Sections 4-6.

Remark 1.2. This observation can also be found in [23, Theorem 2.2] for the Laplace equation with $\mathcal{A}=$ id the identity operator. For the Stokes and Navier-Lamé equations, the operators $\mathcal{A}$ are $\frac{\operatorname{dev}}{\mu}$ and $\mathbb{C}^{-1}$ with the operators dev (and $\mu$ ) and $\mathbb{C}^{-1}$ of Sections 5 and 6 .

Since $\left\|\mathcal{A}\left(p_{h}\right)-D \tilde{u}_{h}\right\|_{L^{2}(\Omega)} \lesssim\left\|D_{\mathcal{T}} u_{h}-D \tilde{u}_{h}\right\|_{L^{2}(\Omega)}$ for the aforementioned problems, the second aim of this paper reads

$$
\min _{\tilde{u}_{h} \in V}\left\|D_{\mathcal{T}} u_{h}-D \tilde{u}_{h}\right\|_{L^{2}(\Omega)}^{2} \lesssim \sum_{E \in \mathcal{E}} \sum_{z \in \mathcal{K}(E)} h_{E}\left\|\gamma_{\tau_{E}}\left(\left[D_{\mathcal{T}}\left(\psi_{z} u_{h}\right)\right]\right)\right\|_{L^{2}(E)}^{2}=: \mu^{2}
$$

for the jumps $\left[D_{\mathcal{T}}\left(u_{h} \psi_{z}\right)\right]$ of a discrete nonconforming finite element function $u_{h}$ times a weight-function $\psi_{z}$ across some side $E$ with vertex $z$; details on the notation can be found in Section 3. The second main result (Theorem 3.1) holds for all piecewise gradients and employs a localisation argument with the (modified) hat functions $\left(\psi_{z}: z \in \mathcal{K}\right)$ of the free nodes $\mathcal{K}$.

Then, a summary of these two aims (See, Theorem 2.1 and Theorem 3.1) and (1.7) concludes the main result of this paper

$$
\left\|p-p_{h}\right\|_{L} \lesssim \eta+\mu+\operatorname{osc}(g)
$$

for the unified a posterior error estimate of the nonconforming finite element methods with (H1)-(H3) of Section 2 and for all aforemented problems. This conclusion will be exhibited for each problem in Sections 4-6, what is left is to check the well-posdeness of (1.3)-(1.4) (or (1.2)) for each problem and (H1)-(H3) for each nonconforming finite element scheme; see Sections 4-6 for further details. 
The rest of this paper is organized as follows. While Section 2-3 treat general assertions on (1.10)-(1.11) where condition (1.9) is substituted by (H1)-(H3), Sections 4-6 conclude this paper with particular model examples in $2 D$ (and some in $3 D$ ) with first reliability proofs for many nonstandard finite element error estimates.

Throughout this paper, $V_{h}^{c}$ and $V_{h}^{n c}$ denote conforming and nonconforming finite element spaces based on a regular triangulation $\mathcal{T}$ of $\Omega ; \nu$ denotes the normal unit vector along the boundary $\partial \Omega ; \tau$ denotes the tangent vector along the boundary for 2D. Colon ":" denotes the scalar product in $\mathbb{R}^{m \times n}$, i.e., $A: B:=\sum_{j=1}^{m} \sum_{k=1}^{n} A_{j k} B_{j k}$.

\section{RELIABILITY CONTROL OF THE EQUILIBRIUM RESIDUAL}

This section establishes an explicit residual-based error estimate (1.8) for a class of nonstandard finite element schemes.

Let $V=H_{0}^{1}(\Omega)^{m}$ and $L=L^{2}\left(\Omega ; \mathbb{R}^{m \times n}\right)$ denote standard Sobolev and Lebesgue spaces on some bounded Lipschitz domain $\Omega$ in $\mathbb{R}^{n}$ with a piecewise flat boundary $\Gamma$. Suppose that the closure $\bar{\Omega}$ is covered exactly by a regular triangulation $\mathcal{T}$ of $\bar{\Omega}$ into (closed) triangles or parallelograms in $2 D$, tetrahedrons or parallelepipeds in $3 D$ (or other unions of simplices). It is assumed, that

$$
\bar{\Omega}=\cup \mathcal{T} \quad \text { and } \quad\left|T_{1} \cap T_{2}\right|=0 \quad \text { for } T_{1}, T_{2} \in \mathcal{T} \quad \text { with } T_{1} \neq T_{2},
$$

where $|\cdot|$ denotes the volume (as well as the modulus of a vector etc. where there is no real risk of confusion). The remaining assumptions on the shape regularity of $\mathcal{T}$ are hidden in the following abstract conditions.

(H1) There exists a Clement-type operator $J: V \rightarrow V_{h}^{c}$ into some (conforming) subspace $V_{h}^{c} \subseteq V$ of $\mathcal{T}$-piecewise smooth functions such that, for all $v \in V$ and $T \in \mathcal{T}$

$$
\begin{array}{r}
h_{T}^{-1}\|v-J v\|_{L^{2}(T)}+h_{T}^{-1 / 2}\|v-J v\|_{L^{2}(\partial T)} \\
+\|D(v-J v)\|_{L^{2}(T)} \lesssim\|D v\|_{L^{2}\left(\omega_{T}\right)},
\end{array}
$$

with some neighbourhood $\omega_{T}$ of $\mathrm{T}$ such that $\left(\omega_{T}: T \in \mathcal{T}\right)$ has finite overlap

$$
\max _{x \in \bar{\Omega}} \operatorname{card}\left\{T \in \mathcal{T}: x \in \omega_{T}\right\} \lesssim 1
$$

(H2) There exists a nonconforming space $V_{h}^{n c} \subseteq L^{2}(\Omega)^{m}$ of $\mathcal{T}$-piecewise smooth and, in general, discontinuous functions $V_{h}^{n c} \subseteq H^{1}(\mathcal{T})^{m} \not \subset V$. Given distinct $T_{1}, T_{2} \in$ $\mathcal{T}$, their intersection $T_{1} \cap T_{2}$ has zero volume measure by (2.1) but possibly a positive surface measure $h_{E}$. The set of all interior (edges or faces etc.) $T_{1} \cap T_{2}=E$ is denoted by $\mathcal{E}$. For any $v_{h} \in V_{h}^{n c}$, the jump

$$
\left[v_{h}\right]_{E}(x):=\left(\left.v_{h}\right|_{T_{2}}\right)(x)-\left(\left.v_{h}\right|_{T_{1}}\right)(x) \quad \text { for } x \in E .
$$

across $E \in \mathcal{E}$ with $E=T_{1} \cap T_{2}$ is fixed up to the sign which results from the orientation of the unit vector $\nu_{E}$ on $E$ (e.g. $\nu_{E}$ points outward of $T_{2}$ ). The shape regularity of $\mathcal{T}$ and $\mathcal{E}$ is described by the assumption

$$
h_{E} \approx h_{T} \approx \operatorname{diam}\left(\omega_{T}\right) \quad \text { for all } E \in \mathcal{E}, T \in \mathcal{T} \text { with } E \cap T \neq \emptyset .
$$


Remark 2.1. The trace inequality yields, for $v \in V$ and $T \in \mathcal{T}[44,18]$,

$$
\|v\|_{L^{2}(\partial T)} \lesssim h_{T}^{-1 / 2}\|v\|_{L^{2}(T)}+h_{T}^{1 / 2}\|D v\|_{L^{2}(T)} .
$$

Hence the trace term with $L^{2}(\partial T)$ in (2.2) is estimated by the other two $L^{2}(T)$ norms. More over, if $\mathcal{E}(T)$ denotes the set of all $E$ with $E \subseteq \partial T$, the shape regularity (2.5) shows that

$$
\sum_{E \in \mathcal{E}(T)} h_{E}^{-1}\|v-J v\|_{L^{2}(E)}^{2} \lesssim\|D v\|_{L^{2}\left(\omega_{T}\right)} .
$$

Remark 2.2. The conforming functions are given as those with vanishing jumps, i.e., $v_{h} \in V_{h}^{c}$ implies $\left[v_{h}\right]_{E}=0$ for all $E \in \mathcal{E}$.

The aforementioned standard assumptions are typical in finite element simulations. The innovative condition on the nonstandard finite element space $V_{h}^{n c}$ and the conforming counterpart $V_{h}^{c}$ of (H1)-(H2) is the following.

(H3) There exists some operator $\Pi: V_{h}^{c} \rightarrow V_{h}^{n c}$ such that, for all $v_{h} \in V_{h}^{c}$ and all $T \in \mathcal{T}$, there holds

$$
\left\|\nabla\left(\Pi v_{h}\right)\right\|_{L^{2}(T)} \lesssim\left\|\nabla v_{h}\right\|_{L^{2}\left(\omega_{T}\right)} \text { and } \int_{T} v_{h} d x=\int_{T} \Pi v_{h} d x .
$$

Moreover, for some given discrete approximation $p_{h} \in L^{2}\left(\Omega ; \mathbb{R}^{m \times n}\right)$ and the $\mathcal{T}$ piecewise gradient $D_{\mathcal{T}}$, there holds

$$
\int_{\Omega} p_{h}: D_{\mathcal{T}} v_{h} d x=\int_{\Omega} p_{h}: D_{\mathcal{T}}\left(\Pi v_{h}\right) d x .
$$

A direct consequence of $(2.8)$ is

$$
h_{T}^{-1}\left\|v_{h}-\Pi v_{h}\right\|_{L^{2}(T)} \lesssim\left\|D v_{h}\right\|_{L^{2}\left(\omega_{T}\right)} \text { for all } T \in \mathcal{T} .
$$

Given $g \in L^{2}(\Omega)^{m}$ and $p_{h}$ as above, the residual $\mathcal{R}_{e} s_{V} \in V^{*}$ is, for $v \in V+V_{h}^{n c} \subset$ $L^{2}\left(\Omega, \mathbb{R}^{m \times n}\right)$ defined by

$$
\mathcal{R}_{e} s_{V}(v):=\int_{\Omega} g \cdot v d x-\int_{\Omega} p_{h}: D_{\mathcal{T}} v d x
$$

The residual is supposed to stem from a nonstandard finite element scheme with $V_{h}^{n c}$ and hence

$$
\operatorname{Res}_{V}\left(v_{h}\right)=0 \quad \text { for all } v_{h} \in V_{h}^{n c} .
$$

With the abbreviation $g_{T}:=|T|^{-1} \int_{T} g(x) d x \in \mathbb{R}^{m}$, the data oscillation reads

$$
\operatorname{osc}(g):=\left(\sum_{T \in \mathcal{T}} h_{T}^{2}\left\|g-g_{T}\right\|_{L^{2}(T)}^{2}\right)^{1 / 2} .
$$

Under the assumptions of (H1)-(H3), the residual-based error estimator

$$
\eta:=\left(\sum_{T \in \mathcal{T}} h_{T}^{2}\left\|g+\operatorname{div} p_{h}\right\|_{L^{2}(T)}^{2}\right)^{1 / 2}+\left(\sum_{E \in \mathcal{E}} h_{E}\left\|\left[p_{h}\right]_{E} \cdot \nu_{E}\right\|_{L^{2}(E)}^{2}\right)^{1 / 2}
$$

is reliable in the following sense.

Theorem 2.1. There holds $\left\|\mathcal{R} e s_{V}\right\|_{V^{*}} \lesssim \eta+\operatorname{osc}(g)$. 
Proof. Given any $v \in V$ with $\Pi J v \in V_{h}^{n c},(2.12)$ leads to

$$
\operatorname{Res}_{V}(v)=\int_{\Omega} g \cdot(v-\Pi J v) d x-\int_{\Omega} p_{h}: D_{\mathcal{T}}(v-\Pi J v) d x .
$$

An elementwise integration by parts and a careful re-arrangement of boundary pieces leads to

$$
\begin{aligned}
\int_{\Omega} p_{h}: D(v-J v) d x & =-\int_{\Omega}\left(\operatorname{div}_{\mathcal{T}} p_{h}\right) \cdot(v-J v) d x \\
& +\sum_{E \in \mathcal{E}} \int_{E}\left[p_{h}\right] \cdot \nu_{E}(v-J v) d s .
\end{aligned}
$$

The combination of the two identities with (2.9), i.e., $\int_{\Omega} p_{h}: D_{\mathcal{T}}(J v-\Pi J v) d x=0$, where $v_{h}$ is replaced by $J v \in V_{h}^{c}$, reads

$$
\begin{aligned}
\operatorname{Res}_{V}(v)= & \int_{\Omega}\left(g+\operatorname{div}_{\mathcal{T}} p_{h}\right) \cdot(v-J v) d x+\int_{\Omega} g \cdot(J v-\Pi J v) d x \\
& -\sum_{E \in \mathcal{E}} \int_{E}\left[p_{h}\right] \cdot \nu_{E}(v-J v) d s=: I_{1}+I_{2}+I_{3} .
\end{aligned}
$$

The first integral $I_{1}$ on the right-hand side is controlled with (2.2)-(2.3), Hölder inequality and Cauchy inequalities. This leads to

$$
I_{1} \lesssim\left(\sum_{T \in \mathcal{T}} h_{T}^{2}\left\|g+\operatorname{div} p_{h}\right\|_{L^{2}(T)}^{2}\right)^{1 / 2}\|D v\|_{L^{2}(\Omega)} .
$$

The second term $I_{2}$ requires (2.8), (2.10) and (2.13). This yields

$$
\begin{aligned}
I_{2} & =\sum_{T \in \mathcal{T}} \int_{T} g \cdot(J v-\Pi J v) d x \\
& =\sum_{T \in \mathcal{T}} \int_{T}\left(g-g_{T}\right) \cdot(J v-\Pi J v) d x \\
& \leqslant \sum_{T \in \mathcal{T}} h_{T}\left\|g-g_{T}\right\|_{L^{2}(T)} h_{T}^{-1}\|J v-\Pi J v\|_{L^{2}(T)} \\
& \lesssim \operatorname{osc}(g)\left(\sum_{T \in \mathcal{T}}\left\|D v_{h}\right\|_{L^{2}\left(\omega_{T}\right)}^{2}\right)^{1 / 2} \\
& \lesssim \operatorname{osc}(g)\|D v\|_{L^{2}(\Omega)} .
\end{aligned}
$$

Standard arguments with (2.1) - (2.3) and (2.7) control the last term

$$
\begin{aligned}
I_{3} & \leqslant \sum_{E \in \mathcal{E}} h_{E}^{1 / 2}\left\|\left[p_{h}\right]_{E} \cdot \nu_{E}\right\|_{L^{2}(E)} h_{E}^{-1 / 2}\|v-J v\|_{L^{2}(E)} \\
& \lesssim\left(\sum_{E \in \mathcal{E}} h_{E}\left\|\left[p_{h}\right]_{E} \cdot \nu_{E}\right\|_{L^{2}(E)}^{2}\right)^{1 / 2}\|D v\|_{L^{2}(\Omega)} .
\end{aligned}
$$

Altogether, there follows the assertion

$$
\mathcal{R}_{e s_{V}}(v)=I_{1}+I_{2}+I_{3} \lesssim(\eta+\operatorname{osc}(g))\|D v\|_{L^{2}(\Omega)} .
$$




\section{Reliability CONTROL OF THE CONSISTENCY RESIDUAL}

This section establishes a general control of the consistency residual (1.11). Given $u_{h} \in V_{h}^{n c}$ with $D_{\mathcal{T}} u_{h} \in L^{2}\left(\Omega ; \mathbb{R}^{m \times n}\right)$ and the conforming finite element space $V_{h}^{c}$ from (H1)-(H3), let $\left(\psi_{z}: z \in \mathcal{K}\right)$ denote a Lipschitz continuous partition of unity,

$$
\sum_{z \in \mathcal{K}} \psi_{z}=1 \quad \text { in } \Omega
$$

Moreover, for any $z \in \mathcal{K}$, suppose that, $\psi_{z}$ vanishes outside an open and connected set $\Omega_{z} \subseteq \Omega$

$$
\operatorname{supp} \psi_{z} \subseteq \bar{\Omega}_{z} \quad \text { and } \quad \max _{x \in \bar{\Omega}} \operatorname{card}\left\{z \in \mathcal{K}: x \in \Omega_{z}\right\} \lesssim 1
$$

Given $z \in \mathcal{K}$, let $\mathcal{E}(z):=\left\{E \in \mathcal{E}:\left.\psi_{z}\right|_{E} \not \equiv 0\right\}$ denote the set of edges, where $\psi_{z}$ is nonvanishing. For any edge $E$ let $\mathcal{K}(E)$ denote the set of all $z \in \mathcal{K}$ with $E \in \mathcal{E}(z)$. The tangential component of a vector $v \in \mathbb{R}^{n}$ is defined as

$$
\gamma_{\tau_{E}}(v):=\left\{\begin{array}{l}
v \cdot \tau_{E} \text { if } n=2 \\
v \times \nu_{E} \text { if } n=3
\end{array}\right.
$$

The general estimator

$$
\mu:=\left(\sum_{E \in \mathcal{E}} \sum_{z \in \mathcal{K}(E)} h_{E}\left\|\gamma_{\tau_{E}}\left(\left[D_{\mathcal{T}}\left(\psi_{z} u_{h}\right)\right]\right)\right\|_{L^{2}(E)}^{2}\right)^{1 / 2}
$$

is reliable in the following sense.

Theorem 3.1. For $n=2$, 3, there holds $\min _{\tilde{u}_{h} \in V}\left\|D_{\mathcal{T}} u_{h}-D \tilde{u}_{h}\right\|_{L^{2}(\Omega)} \lesssim \mu$.

Remark 3.1. In the examples below, $0 \leq \psi_{z} \leq 1$ is a finite sum of hat functions and continuous such that $\gamma_{\tau_{E}}\left(\left[D_{\mathcal{T}}\left(\psi_{z} u_{h}\right)\right]\right)=\gamma_{\tau_{E}}\left(D \psi_{z}\right)\left[u_{h}\right]+\psi_{z} \gamma_{\tau_{E}}\left(\left[D_{\mathcal{T}} u_{h}\right]\right)$. Moreover, the polynomial $\left[u_{h}\right]$ has some zero on $E$ and allows an estimate

$$
\left\|\left[u_{h}\right]\right\|_{L^{2}(E)} \lesssim h_{E}\left\|\gamma_{\tau_{E}}\left(\left[D_{\mathcal{T}} u_{h}\right]\right)\right\|_{L^{2}(E)} .
$$

With $\left\|D \psi_{z}\right\|_{L^{\infty}} \approx h_{E}^{-1}$, one deduces

$$
\mu \lesssim\left(\sum_{E \in \mathcal{E}} h_{E}\left\|\gamma_{\tau_{E}}\left(\left[D_{\mathcal{T}} u_{h}\right]\right)\right\|_{L^{2}(E)}^{2}\right)^{1 / 2}
$$

This estimator is the frequently found version of the consistency error control [36, $37,31,27]$.

Remark 3.2. Theorem 3.1 generalizes [31]. To control the nonconformity, it was assumed therein that

$$
\int_{E}\left[v_{h}\right] d s=0 \text { for } E \in \mathcal{E} \text { and } \int_{E} v_{h} d s=0 \text { for } E \text { on } \partial \Omega \text { for all } v_{h} \in V_{h}^{n c} .
$$

The condition (3.7) is removed in Theorem 3.1 of the present paper.

Proof of Theorem 3.1. Given $z \in \mathcal{K}$ let $a_{z}$ and $b_{z}$ denote the functions of the Helmholtz decomposition of $D_{\mathcal{T}}\left(\psi_{z} u_{h}\right)$, i.e.,

$$
D_{\mathcal{T}}\left(\psi_{z} u_{h}\right)=D a_{z}+\operatorname{curl} b_{z} \in L,
$$


Here $a_{z} \in H_{0}^{1}\left(\Omega_{z}\right), b_{z} \in H^{1}\left(\Omega_{z}\right)^{k}$ with $\int_{\Omega_{z}} b_{z}(x) d x=0$, and $k=1$ for $n=2$ while $k=3$ for $n=3$. Since $\int_{\Omega_{z}} \operatorname{curl} b_{z}: D a d x=0$ for any $a \in H_{0}^{1}\left(\Omega_{z}\right)$,

$$
\begin{aligned}
\left\|\operatorname{curl} b_{z}\right\|_{L^{2}\left(\Omega_{z}\right)}^{2} & =\min _{a \in H_{0}^{1}\left(\Omega_{z}\right)}\left\|D a-D_{\mathcal{T}}\left(\psi_{z} u_{h}\right)\right\|_{L^{2}\left(\Omega_{z}\right)}^{2} \\
& =\int_{\Omega_{z}}\left(\operatorname{curl} b_{z}\right): D_{\mathcal{T}}\left(\psi_{z} u_{h}\right) d x .
\end{aligned}
$$

An elementwise integration by parts followed by $\operatorname{curl}_{\mathcal{T}} D_{\mathcal{T}} \equiv 0$ yields

$$
\begin{aligned}
\int_{\Omega_{z}}\left(\operatorname{curl} b_{z}\right): D_{\mathcal{T}}\left(\psi_{z} u_{h}\right) d x & = \pm \int_{\cup \mathcal{E}(z)} b_{z} \cdot \gamma_{\tau_{E}}\left(\left[D_{\mathcal{T}}\left(\psi_{z} u_{h}\right)\right]\right) d s \\
& \leqslant\left\|\gamma_{\tau_{E}}\left(\left[D_{\mathcal{T}}\left(\psi_{z} u_{h}\right)\right]\right)\right\|_{L^{2}(\bigcup \mathcal{E}(z))}\left\|b_{z}\right\|_{L^{2}(\bigcup \mathcal{E}(z))},
\end{aligned}
$$

where $\mathcal{E}(z):=\left\{E \in \mathcal{E}:\left.\psi_{z}\right|_{E} \not \equiv 0\right\}$. The well-known trace theorem on each element domain $K$, namely

$$
\left\|b_{z}\right\|_{L^{2}(\partial K)} \leqslant h_{K}^{-1 / 2}\left\|b_{z}\right\|_{L^{2}(K)}+h_{K}^{1 / 2}\left\|D b_{z}\right\|_{L^{2}(K)},
$$

leads to the estimate

$$
\left\|b_{z}\right\|_{L^{2}(\bigcup \mathcal{E}(z))} \lesssim h_{z}^{-1 / 2}\left\|b_{z}\right\|_{L^{2}\left(\Omega_{z}\right)}+h_{z}^{1 / 2}\left\|D b_{z}\right\|_{L^{2}\left(\Omega_{z}\right)} .
$$

A Poincaré inequality gives

$$
\left\|b_{z}\right\|_{L^{2}\left(\Omega_{z}\right)} \lesssim h_{z}\left\|D b_{z}\right\|_{L^{2}\left(\Omega_{z}\right)} \lesssim h_{z}\left\|\operatorname{curl} b_{z}\right\|_{L^{2}\left(\Omega_{z}\right)} .
$$

The latter inequality results from the stability of the Helmholtz decomposition [27, 44] with an $h_{z}$-independent constant; it reads $\left\|D b_{z}\right\|_{L^{2}\left(\Omega_{z}\right)}=\left\|\operatorname{curl} b_{z}\right\|_{L^{2}\left(\Omega_{z}\right)}$ in $2 D$. The combination of the proceeding three inequalities leads to

$$
\left\|b_{z}\right\|_{L^{2}(\cup \mathcal{E}(z))} \lesssim h_{z}^{1 / 2}\left\|\operatorname{curl} b_{z}\right\|_{L^{2}\left(\Omega_{z}\right)} .
$$

Since $\left.h_{\mathcal{T}}\right|_{\Omega_{z}} \approx h_{z}:=\operatorname{diam}\left(\Omega_{z}\right), z \in \mathcal{K}$, the aforementioned arguments imply

$$
\left\|D a_{z}-D_{\mathcal{T}}\left(\psi_{z} u_{h}\right)\right\|_{L^{2}\left(\Omega_{z}\right)} \lesssim\left\|h_{\mathcal{E}}^{1 / 2} \gamma_{\tau_{E}}\left(\left[D_{\mathcal{T}}\left(\psi_{z} u_{h}\right)\right]\right)\right\|_{L^{2}(\cup \mathcal{E}(z))} .
$$

Since $\sum_{z \in \mathcal{K}} \psi_{z} \equiv 1$ and $\tilde{u}_{h}:=\sum_{z \in \mathcal{K}} a_{z} \in H_{0}^{1}(\Omega)$, this estimate plus the finite overlap of all $\Omega_{z}$ and $\mathcal{E}(z)$ prove the assertion. In fact,

$$
\begin{aligned}
\left\|D_{\mathcal{T}} u_{h}-D \tilde{u}_{h}\right\|_{L}^{2} & =\left\|\sum_{z \in \mathcal{K}}\left(D_{\mathcal{T}}\left(\psi_{z} u_{h}\right)-D a_{z}\right)\right\|_{L}^{2} \\
& \lesssim \sum_{z \in \mathcal{K}}\left\|D a_{z}-D_{\mathcal{T}}\left(\psi_{z} u_{h}\right)\right\|_{L^{2}\left(\Omega_{z}\right)}^{2} \\
& \lesssim \sum_{z \in \mathcal{K}}\left\|h_{\mathcal{E}}^{1 / 2} \gamma_{\tau_{E}}\left(\left[D_{\mathcal{T}}\left(\psi_{z} u_{h}\right)\right]\right)\right\|_{L^{2}(\cup \mathcal{E}(z))}^{2} \\
& \approx\left(\sum_{E \in \mathcal{E}} \sum_{z \in \mathcal{K}(E)} h_{E}\left\|\gamma_{\tau_{E}}\left(\left[D_{\mathcal{T}}\left(\psi_{z} u_{h}\right)\right]\right)\right\|_{L^{2}(E)}^{2}\right) .
\end{aligned}
$$




\section{Application to Laplace Equation}

This section is devoted to the Poisson problem and its residual-based a posteriori finite element error control. Subsection 4.1 introduces the model problem and Subsection 4.2 some required notations. Subsection 4.3 presents a list of examples. Subsections 4.4-4.5 present the applications of the theory to the mortar and dG finite element methods. Subsection concerns the extension of the present theory to the high-order nonconforming finite element method.

4.1. Model Problem. The Lebesgue and Sobolev spaces $L^{2}(\Omega)$ and $H^{1}(\Omega)$ are defined as usual and

$$
L:=L^{2}(\Omega)^{n} \text { and } V:=H_{0}^{1}(\Omega):=\left\{w \in H^{1}(\Omega): w=0 \text { on } \partial \Omega\right\} .
$$

The gradient operator $\nabla$ maps $V$ into $L$. Given $g \in L^{2}(\Omega)$ let $u \in V$ denote the solution to the Poisson Problem

$$
\Delta u+g=0 \text { in } \Omega \text { and } u=0 \text { on } \partial \Omega .
$$

Then, the flux $p:=\nabla u \in L$ and $u \in V$ satisfy

$$
\begin{aligned}
(A(p, u))(q, v) & :=a(p, q)+b(p, v)+b(q, u) \\
& \stackrel{!}{=}-\int_{\Omega} g v d x \quad \text { for all }(q, v) \in X=L \times V .
\end{aligned}
$$

Throughout this section, (1.1)-(1.7) hold for

$$
a(p, q):=\int_{\Omega} p \cdot q d x \quad \text { and } \quad b(p, v):=-\int_{\Omega} p \cdot \nabla v d x .
$$

The operator $A: X \rightarrow X^{*}$ is bounded, linear, and bijective [23].

4.2. Nonconforming finite element methods and unified a posteriori error estimators. Let $P_{k}(T)$ and $Q_{k}(T)$ denote the space of algebraic polynomials of total and partial degree $\leq k$, respectively, and set $\mathcal{P}_{k}(T)=P_{k}(T)$ and $\mathcal{P}_{k}(T)=Q_{k}(T)$ for a triangle (or tetrahedron) and parallelogram (or parallelepiped), respectively. Define

$$
\begin{aligned}
& \mathcal{P}_{k}(\mathcal{T}):=\left\{v \in L^{2}(\Omega): \forall T \in \mathcal{T},\left.v\right|_{T} \in \mathcal{P}_{k}(T)\right\} \quad \text { for } k=0,1 \\
& \mathcal{S}^{1}(\mathcal{T}):=\mathcal{P}_{1}(\mathcal{T}) \cap C(\bar{\Omega}) \quad \text { and } \quad V_{h}^{c}:=\mathcal{S}_{0}^{1}(\mathcal{T}):=\mathcal{S}^{1}(\mathcal{T}) \cap V
\end{aligned}
$$

Let $\mathcal{N}$ denote the set of nodes (i.e., vertices of elements in $\mathcal{T}$ )u. $h_{\mathcal{T}}$ and $h_{\mathcal{E}}$ denote $\mathcal{T}$ - and $\mathcal{E}$-piecewise constant functions on $\Omega$ and $\cup \mathcal{E}=\cup_{E \in \mathcal{E}} E$ defined by $\left.h_{\mathcal{T}}\right|_{T}:=$ $h_{T}:=\operatorname{diam}(T)$ and $\left.h_{\mathcal{E}}\right|_{E}:=h_{E}:=\operatorname{diam}(E)$ for $T \in \mathcal{T}$ and $E \in \mathcal{E}$. For a given quadrilateral or parallelepiped element $T \in \mathcal{T}, \mathcal{F}_{T}: \hat{T}=[-1,1]^{n} \rightarrow T$ denotes the canonical bilinear transformation.

Let $V_{h}^{n c}$ denote some nonconforming finite element space specified in Table 4.1. For the moment solely suppose that $\nabla_{\mathcal{T}} v_{h} \in L$ for any $v_{h} \in V_{h}^{n c}$, where $\nabla_{\mathcal{T}}$ denote the $\mathcal{T}$-piecewise action of the gradient operator. The finite element solution $u_{h} \in V_{h}^{n c}$ is the unique solution to

$$
\int_{\Omega} \nabla_{\mathcal{T}} u_{h} \cdot \nabla_{\mathcal{T}} v_{h} d x=\int_{\Omega} g v_{h} d x \quad \text { for all } v_{h} \in V_{h}^{n c}
$$




\begin{tabular}{|c|c|c|c|}
\hline picture & name & reference & space \\
\hline$\hat{S}$ & Crouzeix-Raviart & {$[35]$} & $V_{h}^{C R}$ \\
\hline & Wilson & {$[73,64]$} & $V_{h}^{W i l}$ \\
\hline & Han & {$[45]$} & $V_{h}^{\text {Han }}$ \\
\hline & NR (midpoint) & {$[60]$} & $V_{h}^{R T, P}$ \\
\hline & NR (average) & {$[60]$} & $V_{h}^{R T, A}$ \\
\hline & CNR & {$[50]$} & $V_{h}^{C R T}$ \\
\hline & DSSY & {$[40]$} & $V_{h}^{D S S Y}$ \\
\hline
\end{tabular}

TABLE 4.1. Nonconforming Elements for the Laplace Equation (4.2) with (H1)-(H3) and the Error Estimate (4.8).

The aim is to estimate the flux error $p-p_{h}$ for the discrete flux $p_{h}:=\nabla_{\mathcal{T}} u_{h} \in L=$ $L^{2}(\Omega)^{n}$.

For any $\tilde{u}_{h} \in V$ there holds (1.7) for $\mathcal{R} e s_{L} \in L^{*}$ and $\mathcal{R} e s_{V} \in V^{*}$ defined, for all $q \in L$ and $v \in V$, by

$$
\begin{aligned}
\mathcal{R}_{e s}(q) & :=\int_{\Omega} q \cdot\left(\nabla \tilde{u}_{h}-p_{h}\right) d x \text { and } \\
\mathcal{R}_{e} s_{V}(v) & :=-\int_{\Omega} g v d x+\int_{\Omega} p_{h} \cdot \nabla v d x .
\end{aligned}
$$

4.3. Examples. This subsection presents a list of $2 D$ and $3 D$ nonconforming finite element spaces $V_{h}^{n c}$ of Table 4.1 with (H1)-(H3), so that

$$
\left\|p-p_{h}\right\|_{L^{2}(\Omega)} \lesssim \eta+\mu+\operatorname{osc}(g)
$$

with $\eta$ from (2.14), $\mu$ from (3.4), and $\operatorname{osc}(g)$ from (2.13). This list below is not comprehensive. In fact, we conjecture that all known NCFEMs could be analyzed in the present framework. Only the triangular Crouzeix-Raviart element has already been analyzed in [23]. The present unifying theory leads to new error control (4.8) for all nonconforming finite elements of Subsubsections 4.3.2-4.3.6.

4.3.1. The triangular Crouzeix-Raviart element. Based on the regular triangulation $\mathcal{T}$ into simplices, the set of midpoints $\mathcal{M}$ of edges (or faces), the non-conforming Crouzeix-Raviart finite element space reads (in 2D and 3D)

$$
V_{h}^{C R}:=\left\{v \in \mathcal{P}_{1}(\mathcal{T}): v \text { continuous at } \mathcal{M} \cap \Omega \text { and } v=0 \text { at } \mathcal{M} \cap \partial \Omega\right\} .
$$

Since $V_{h}^{C} \subset V_{h}^{C R}$, then there holds (H1)-(H3) with $\Pi=$ id; cf. Section 4 of [31] for proofs. Similar arguments verify (H1)-(H3) in 3D as well; we therefore omit the details. 
4.3.2. The Quadrilateral Wilson element. Let $B$ denote one of the nonconforming quadratic bubble function spaces on the reference element $\hat{T}=[-1,1]^{n}$, i.e.,

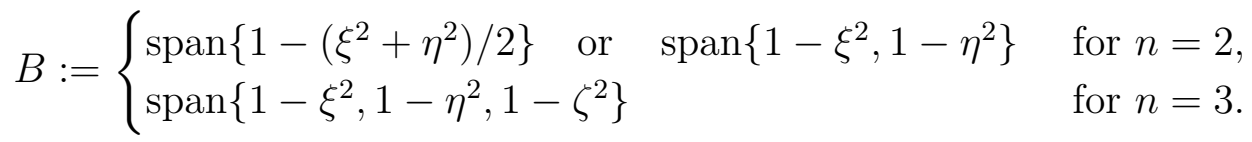

The nonconforming quadrilateral Wilson finite element space $V_{h}^{W i l}[73,64]$ reads

$$
\begin{aligned}
V_{h}^{W i l} & =S^{h} \oplus B^{h} \text { with the factors } \\
S^{h} & :=\left\{v \in H_{0}^{1}(\Omega): \forall T \in \mathcal{T}, \quad \hat{v}=v \circ \mathcal{F}_{T} \in Q_{1}(\hat{T})\right\}, \\
B^{h} & :=\left\{v \in L^{2}(\Omega): \forall T \in \mathcal{T}, \quad \hat{v}=v \circ \mathcal{F}_{T} \in B\right\} .
\end{aligned}
$$

This element is excluded from the analysis of $[31,23]$ since (3.7) is violated. However, there holds (H1)-(H3) with $\Pi=\mathrm{id}$, the proof is immediate since $V_{h}^{c} \subset V_{h}^{W i l}$.

4.3.3. The parallelogram nonconforming Han element. Consider the functional

$$
\mathcal{F}_{E}(v)=|E|^{-1} \int_{E} v d s \text { for all } E \in \mathcal{E}(T) \text { and } T \in \mathcal{T} .
$$

The parametric formulation of rectangular and parallelogram elements of Han [45] is introduced by

$$
\mathcal{Q}_{\mathcal{H}}^{n c}:=\operatorname{span}\left\{1, \xi, \eta, \xi^{2}-\frac{5}{3} \xi^{4}, \eta^{2}-\frac{5}{3} \eta^{4}\right\}
$$

The nonconforming Han finite element space then reads (with $[\cdot]:=\cdot$ along $\partial \Omega$ )

$$
V_{h}^{\text {Han }}:=\left\{v \in L^{2}(\Omega): \forall T \in \mathcal{T},\left.v\right|_{T} \circ \mathcal{F}_{T} \in \mathcal{Q}_{\mathcal{H}}^{n c} \text { and } \forall E \in \mathcal{E}, \mathcal{F}_{E}([v])=0\right\} .
$$

Then there holds (H1)-(H3) with the associated interpolation operator $\Pi$ for $V_{h}^{H a n}$ [45], the proof follows from $\Pi V_{h}^{c}=V_{h}^{C R T} \subset V_{h}^{H a n}$ [50] with $V_{h}^{C R T}$ from Subsubsection 4.3.5 below. Further details for the properties of $\Pi$ can be found in Section 4 of [31], Remark 2.5 and Lemma 3.1 of [50]

4.3.4. The parallelogram nonconforming rotated $Q_{1}$ elements. Rannacher and Turek introduce two types of parallelogram nonconforming elements [60], called the $N R$ elements. The first element $R T A$ uses the average of the function over the edge ( or face) as the local degree of freedom, and the second one RTP uses the value at the midside point ( or midpoint ) of the edge ( or face ) instead. Define

$$
\mathcal{Q}_{\mathcal{R}}^{n c}:= \begin{cases}\operatorname{span}\left\{1, \xi, \eta, \xi^{2}-\eta^{2}\right\} & \text { for } n=2, \\ \operatorname{span}\left\{1, \xi, \eta, \zeta, \xi^{2}-\eta^{2}, \xi^{2}-\zeta^{2}\right\} & \text { for } n=3\end{cases}
$$

then nonconforming space $V_{h}^{R T, A}$ is defined in (4.13) with $\mathcal{Q}_{\mathcal{H}}^{n c}$ replaced by $\mathcal{Q}_{\mathcal{R}}^{n c}$, and $V_{h}^{R T, P}$ is defined in $(4.9)$ with $\mathcal{P}_{1}(\mathcal{T})$ replaced by $\mathcal{Q}_{\mathcal{R}}^{n c}$.

For $2 D$, following a similar argument for the Han element, one proves that the average version element satisfies (H1)-(H3) with the canonical interpolation operator $\Pi$ for $V_{h}^{R T, A}$; [31] contains further details. 
The midside point version element is not included in [31] since the condition (3.7) is violated by this element. However, there holds equally (H1)-(H3) for it with the canonical interpolation operator $\Pi$ of $V_{h}^{R T, P}$. In fact, we have

$$
\Pi V_{h}^{c}=V_{h}^{C R T} \subset V_{h}^{R T, P},
$$

and $V_{h}^{C R T}$ contains the linear part of $V_{h}^{c}$, and only the nonlinear part is excluded [50]. With this fact, (H3) follows from straight forward investigations.

For $3 \mathrm{D}$, define the local interpolation operator $\Pi_{T}: H^{1}(T) \rightarrow \mathcal{Q}_{\mathcal{R}}^{n c} \circ \mathcal{F}_{T}^{-1}$ by

$$
\mathcal{F}_{E}\left(\Pi_{T} v\right)=\mathcal{F}_{E}(v) \text { for } E \in \mathcal{E}(T) \text { for all } v \in H^{1}(T) .
$$

Since $\mathcal{F}_{\hat{E}}(v)=0$ for $v=\xi \eta, \xi \zeta, \eta \zeta, \xi \eta \zeta$ with $\hat{E} \in \mathcal{E}(\hat{T})$, we conclude for any $v=$ $a_{0}+a_{1} \xi+a_{2} \eta+a_{3} \zeta+a_{4} \xi \eta+a_{4} \xi \zeta+a_{6} \eta \zeta+a_{7} \xi \eta \zeta$ that

$$
\Pi_{T} v=a_{0}+a_{1} \xi+a_{2} \eta+a_{3} \zeta
$$

with some interpolation constants $a_{0}, \ldots, a_{7}$. The global interpolation operator $\Pi$ is defined by $\left.\Pi\right|_{T}=\Pi_{T}$ for any $T \in \mathcal{T}$. Then (H1)-(H3) eventually follows from (4.17).

Remark 4.1. The analysis does not cover the non-parametric variant of this element except on parallelogram meshes.

4.3.5. The parallelogram constrained nonconforming rotated $Q_{1}$ elements. The constrained rotated nonconforming finite element (referred to as $C N R$ element) introduced in [50] is obtained by enforcing a constraint on the $N R$ element on each element for $2 D$. The space of the $C N R$ element reads

$$
\begin{aligned}
V_{h}^{C R T}:=\left\{v \in V_{h}^{R T, A}: \forall T \in \mathcal{T}, \int_{E_{1}} v d s+\int_{E_{3}} v d s=\int_{E_{2}} v d s+\int_{E_{4}} v d s\right. \\
\left.\quad \text { with }\left\{E_{1}, \cdots, E_{4}\right\}=\mathcal{E}(T) \text { numbered counterclockwise }\right\} .
\end{aligned}
$$

For rectangular and parallelogram meshes, the element is equivalent to the $P_{1^{-}}$ quadrilateral element of [59]. Then there holds (H1)-(H3) with the interpolation operator $\Pi$ of $V_{h}^{C R T}$. The proof follows from the argument for the $N R$ element with the midside point version. We refer to Section 4 of [31] for more details. The goal-oriented error control of this element is given in [43].

4.3.6. The parallelogram DSSY elements. The DSSY element is obtained by introducing on the reference element [40] with $\theta_{1}(t)=t^{2}-\frac{5}{3} t^{4}$ and $\theta_{2}(t)=t^{2}-\frac{25}{6} t^{4}+\frac{7}{2} t^{6}$ and

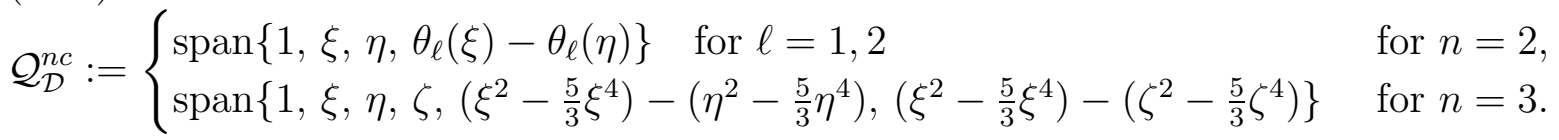

The nonconforming finite element spaces $V_{h}^{D S S Y}$ are defined as in (4.13) with $\mathcal{Q}_{\mathcal{H}}^{n c}$ replaced by $\mathcal{Q}_{\mathcal{D}}^{n c}$. There holds (H1)-(H3) with the interpolation operator $\Pi$ of $V_{h}^{D S S Y}$, cf. the proof in Section 4 of [31] for 2D. Arguments similar to those of Subsection 4.3.4 verify (H1)-(H3) for 3D. 
Remark 4.2. The parallelogram nonconforming element of [57] can also be analyzed by this unifying theory.

4.4. Comments on mortar finite element methods. Another class of nonconforming FEM is known as mortar FEM $[13,14]$ where the continuity of $u_{h}$ over the common side of two subdomains $K^{-}$and $K^{+}$in some locally quasi-uniform regular decomposition $\mathcal{T}_{H}$ of $\Omega$ into triangles is enforced by Lagrange multipliers. The a posteriori error estimates with the saturation assumptions are presented in $[15,74]$. A more general one is analyzed in [12]. For the ease of the discussion, suppose that $n=2$ and that the partition $\mathcal{T}_{h}$ is obtained from $\mathcal{T}_{H}$ by refining some of the triangles in $\mathcal{T}_{H}$ by some finite number $\leq k$ of successive red-refinements (i.e., cutting a triangle into 4 congruent subtriangles by connecting its edges' midpoints) so that the ratio of the diameters of two neighbouring triangles with adjusted edges is bounded by $2^{-k}$. Notice that $(2.5)$ holds for all edges $E$ of $T$ while the equivalence with $\omega_{T}$ depends on $k$.

Let $V_{h}^{n c}$ be the mortar finite element space with respect to $\mathcal{T}_{h}$ as in [12]. With $V_{h}^{c}:=V \cap P_{1}\left(\mathcal{T}_{H}\right)$ one can prove $(\mathrm{H} 1)$ by along the lines of [24]. Since $V_{h}^{c} \subseteq V_{h}^{n c}$, (H3) holds for $\Pi=$ id. Then, Theorem 2.1 reads

$$
\|\operatorname{Res}\|_{V^{*}}^{2} \lesssim \sum_{T \in \mathcal{T}_{h}} H_{T}^{2}\left\|g+\operatorname{div} p_{h}\right\|_{L^{2}(T)}^{2}+\sum_{E \in \mathcal{E}} H_{E}\left\|\left[p_{h}\right] \cdot \nu_{E}\right\|_{L^{2}(E)}^{2}
$$

for $H_{T}:=\max \left\{\operatorname{diam}(K): T \subseteq K \in \mathcal{T}_{H}\right\}$ and $H_{E}:=\max \{\operatorname{diam}(K): E \subset \partial K, K \in$ $\left.\mathcal{T}_{H}\right\}$. Moreover, Theorem 3.1 yields ( with $\mathcal{T}=\mathcal{T}_{h}$ etc.)

$$
\min _{\tilde{u}_{h} \in V}\left\|\operatorname{Res}_{L}\right\|_{L^{*}}^{2} \lesssim \sum_{E \in \mathcal{E}} \sum_{z \in \mathcal{K}(E)} H_{E} \| \gamma_{\tau_{E}}\left(\left[D_{\mathcal{T}}\left(\left(\psi_{z} u_{h}\right)\right]\right) \|_{L^{2}(E)}^{2} .\right.
$$

Therein, $\psi_{z}$ is the partition of unity based with respect to $\mathcal{T}_{H}$ and $\left\|H_{E} D \psi_{z}\right\|_{L^{\infty}} \approx 1$. 2D)

This reliability error estimate is essential Theorem 3.4 in [12]. In fact, since (in

$$
\frac{\partial}{\partial s}\left[\psi_{z} u_{h}\right]=\left(\frac{\partial}{\partial s} \psi_{z}\right)\left[u_{h}\right]+\psi_{z}\left[\partial u_{h} / \partial s\right]
$$

there holds (with an inverse estimate $\left\|\partial\left[u_{h}\right] / \partial s\right\|_{L^{2}(E)} \lesssim H_{E}^{-1}\left\|\left[u_{h}\right]\right\|_{L^{2}(E)}$ ) that

$$
\begin{aligned}
H_{E}\left\|\frac{\partial}{\partial s}\left[\psi_{z} u_{h}\right]\right\|_{L^{2}(E)}^{2} & \lesssim H_{E} H_{T}^{-2}\left\|\left[u_{h}\right]\right\|_{L^{2}(E)}^{2}+H_{E} \cdot\left\|\left[\partial u_{h} / \partial s\right]\right\|_{L^{2}(E)}^{2} \\
& \lesssim\left(H_{E} H_{T}^{-2}+H_{E}^{-1}\right)\left\|\left[u_{h}\right]\right\|_{L^{2}(E)}^{2} \lesssim H_{E}^{-1}\left\|\left[u_{h}\right]\right\|_{L^{2}(E)}^{2} .
\end{aligned}
$$

Altogether, the upper bounds for (1.7) with $p_{h}:=D_{\mathcal{T}} u_{h}$ and $p=\nabla u$ reads

$$
\left\|p-p_{h}\right\|_{L^{2}(\Omega)} \lesssim \sum_{T \in \mathcal{T}_{h}} H_{T}^{2}\left\|g+\operatorname{div} p_{h}\right\|_{L^{2}(T)}^{2}+\sum_{E \in \mathcal{E}} H_{E}\left\|\left[p_{h}\right] \cdot \nu_{E}\right\|_{L^{2}(E)}^{2}+\sum_{E \in \mathcal{E} \cup \mathcal{E}_{\partial \Omega}} H_{E}^{-1}\left\|\left[u_{h}\right]\right\|_{L^{2}(E)}^{2} .
$$

Therein, $\mathcal{E}_{\partial \Omega}$ denote the set of edges on the boundary $\partial \Omega$. Notice $\left[u_{h}\right]=0$ on edges interior to $T \in \mathcal{T}_{H}$.

In comparison to [12, Theorem 3.4], the factor $2^{-k}$ therein is hidden herein the mesh-sizes $H_{T}, H_{E}$. 
4.5. Comments on discontinuous Galerkin methods. The feature for the discontinous Galerkin(abbreviated dG hereafter) methods [4, 38, 71, 5, 7, 8, 52] lies in that the trial and test spaces consist of piecewise discontinuous polynomials. A posteriori error estimates for $\mathrm{dG}$ type methods are considered in [53, 62, 61, 21, 9, 48] for second order elliptic problems, in [46] for the Stokes problem, and in [47, 72] for plane elasticity. This subsection comments on the extension of the unifying theory to dG FEM. For any $v_{h} \in \mathcal{P}_{k}(\mathcal{T})$, the average across $E=T_{1} \cap T_{2}$ reads

$$
<v>_{E}(x):=1 / 2\left(\left(\left.v_{h}\right|_{T_{1}}\right)(x)+\left(\left.v_{h}\right|_{T_{2}}\right)(x)\right) \quad \text { for } x \in E .
$$

With some appropriately chosen constant $\gamma$, the modified bilinear form is defined as

$$
\begin{aligned}
a_{h}^{\gamma}\left(u_{h}, v_{h}\right):= & \sum_{T \in \mathcal{T}} \int_{T} \nabla u_{h} \cdot \nabla v_{h} d x+\gamma \sum_{E \in \mathcal{E}} h_{E}^{-1} \int_{E}\left[u_{h}\right]_{E}\left[v_{h}\right]_{E} d s \\
& -\sum_{E \in \mathcal{E}} \int_{E}\left(<\nabla_{h} u_{h}>_{E} \cdot \nu_{E}\left[v_{h}\right]_{E}+<\nabla_{h} v_{h}>_{E} \cdot \nu_{E}\left[u_{h}\right]_{E}\right) d s \\
& -\sum_{E \subset \partial \Omega} \int_{E}\left(\nabla_{h} u_{h} \cdot \nu_{E} v_{h}+\nabla_{h} v_{h} \cdot \nu_{E} u_{h}\right) d s+\gamma \sum_{E \in \mathcal{E}} h_{E}^{-1} \int_{E} u_{h} v_{h} d s
\end{aligned}
$$

for any $u_{h}, v_{h} \in \mathcal{P}_{k}(\mathcal{T})+H_{0}^{1}(\Omega)$. This is the symmetric dG method from [7, 8, 52, 53]. The discontinuous Galerkin solution $u_{h} \in \mathcal{P}_{k}(\mathcal{T})$ is characterized by

$$
a_{h}^{\gamma}\left(u_{h}, v_{h}\right)=\left(g, v_{h}\right)_{L^{2}(\Omega)} \text { for any } v_{h} \in \mathcal{P}_{k}(\mathcal{T}) .
$$

From $V_{h}^{c} \subset \mathcal{P}_{k}(\mathcal{T})$, there holds $(\mathrm{H} 3)$ with $\Pi=\mathrm{id}$. Theorem 3.1 yields

$$
\min _{\tilde{u}_{h} \in V}\left\|\operatorname{Res}_{L}\right\|_{L^{*}}^{2} \lesssim \sum_{E \in \mathcal{E}} \sum_{z \in \mathcal{K}(E)} h_{E} \| \gamma_{\tau_{E}}\left(\left[D_{\mathcal{T}}\left(\left(\psi_{z} u_{h}\right)\right]\right) \|_{L^{2}(E)}^{2} .\right.
$$

To bound $\left\|\mathcal{R} e s_{V}\right\|_{V^{*}}$, let $v \in V$ and deduce

$$
\begin{aligned}
\operatorname{Res}_{V}(v)= & -\int_{\Omega} g v d x+\int_{\Omega} \nabla_{\mathcal{T}} u_{v} \cdot \nabla v d x \\
= & -\int_{\Omega} g v d x+\int_{\Omega} \nabla_{\mathcal{T}} u_{v} \cdot \nabla v d x \\
& -\sum_{E \in \mathcal{E}} \int_{E}<\nabla_{h} u_{h}>_{E} \cdot \nu_{E}[v]_{E} d s+\gamma \sum_{E \in \mathcal{E}} h_{E}^{-1} \int_{E}\left[u_{h}\right]_{E}[v]_{E} d x \\
& -\sum_{E \subset \partial \Omega} \int_{E}<\nabla_{h} u_{h}>_{E} \cdot \nu_{E}\left[v_{h}\right]_{E} d s+\gamma \sum_{E \subset \partial \Omega} h_{E}^{-1} \int_{E} u_{h} v d s .
\end{aligned}
$$

It follows from $J v \in V \cap \mathcal{P}_{k}(\mathcal{T})$ that

$$
\left\|\mathcal{R} e s_{V}\right\|_{V^{*}} \lesssim \eta+\operatorname{osc}(g)+\left(\sum_{E \in \mathcal{E}} h_{E}^{-1}\left\|\left[u_{h}\right]_{E}\right\|_{L^{2}(E)}^{2}+\sum_{E \subset \partial \Omega} h_{E}^{-1}\left\|u_{h}\right\|_{L^{2}(E)}^{2}\right)^{1 / 2}
$$

Remark 4.3. A combination of the above estimates for $\left\|\mathcal{R} e s_{V}\right\|_{V^{*}}$ and $\min _{\tilde{u}_{h} \in V}\left\|\mathcal{R} e s_{L}\right\|_{L^{*}}$ with (1.7) recovers the estimate

$$
\left\|p-p_{h}\right\|_{L^{2}(\Omega)} \lesssim\left\|\mathcal{R} e s_{V}\right\|_{V^{*}}+\min _{\tilde{u}_{h} \in V}\left\|\mathcal{R} e s_{L}\right\|_{L^{*}}
$$


which appeared in Theorem 3.1 of [9] and Theorem 3.1 from [53] without the assumption $u \in H^{2}(\Omega)$. Where $p=\nabla u$ and $p_{h}=\nabla_{\mathcal{T}} u_{h}$.

Remark 4.4. For brevity, we only consider the a posteriori error estimate of the symmetric dG methods for the Poisson equation, the analysis with corresponding modifications can equally apply to the Stokes problem in Section 5, and the elasticity in Section 6. In particular, this yields the a posteriori error control from Theorem 4.1 of [72] for the plane elasticity, and Theorem 3.1 of [46] for the Stokes problem. Moreover, the unifying theory can be generalized to other $\mathrm{dG}$ methods reviewed in $[5]$.

4.6. Comments on high-order nonconforming schemes. In this paper, we focus on the first-order nonconforming finite element method. The present unifying theory can be extended to high-order nonconforming finite element methods with the corresponding modifications in (H1)-(H3). In fact, Theorem 3.1 holds equally for all nonstandard finite element methods. We only need to modify the the conforming space $V_{h}^{c}$ in (H1) and (H3) and its associated Clemént interpolation operator. For instance, (H3) reads

$$
\int_{T} \Pi v_{h} q d x=\int_{T} v_{h} q d x \text { for any } q \in P_{k-1}(T) \text { and for any } v_{h} \in V_{h}^{c} .
$$

\section{Applications to the Stokes Problem}

5.1. The Stokes Problem. The unsymmetric formulation of the Stokes problem reads: Given $g \in L^{2}(\Omega)^{n}$ seek $(u, p) \in H_{0}^{1}(\Omega)^{n} \times L_{0}^{2}(\Omega)$, such that for all $(v, q) \in$ $H_{0}^{1}(\Omega)^{n} \times L_{0}^{2}(\Omega)$,

$$
\mu \int_{\Omega} D u: D v d x-\int_{\Omega} p \operatorname{div} v d x-\int_{\Omega} q \operatorname{div} u d x=\int_{\Omega} g \cdot v d x .
$$

Here, $L_{0}^{2}(\Omega):=\left\{q \in L^{2}(\Omega): \int_{\Omega} q d x=0\right\} \equiv L^{2}(\Omega) / \mathbb{R}$ fixes a global additive constant in the pressure $p$ (note that $p$ is not the flux from the previous section). The unique existence of solution to (5.1) is well known. Set

$a(\sigma, \tau):=\int_{\Omega} \frac{1}{\mu} \operatorname{dev} \sigma: \operatorname{dev} \tau d x$ for all $\sigma, \tau \in L:=\left\{\tau \in L^{2}\left(\Omega, \mathbb{R}^{n \times n}\right), \int_{\Omega} \operatorname{tr} \tau d x=0\right\}$.

The deviatoric-part operator dev is defined as

$$
\operatorname{dev} F=F-(\operatorname{tr}(F) / n) \text { id } \quad \text { for any } F \in \mathbb{R}^{n \times n} .
$$

with $\operatorname{tr}(F)=F_{11}+\cdots+F_{n n}$. It is known that the operator $A: X=L \times V \rightarrow X^{*}$, defined for $(\sigma, u) \in X$ by

$$
(A(\sigma, u))(\tau, v):=a(\sigma, \tau)-(\sigma, D v)_{L^{2}(\Omega)}-(\tau, D u)_{L^{2}(\Omega)}
$$

is a linear, bounded and bijective, cf. e.g., [23]. 
5.2. Nonconforming finite element methods and unified a posteriori error estimators. Given some nonconforming finite element space $V_{h}^{n c}$ for $V:=H_{0}^{1}(\Omega)^{n}$ and $\mathcal{Q}_{h} \subset L_{0}^{2}(\Omega)$, the finite element solution $\left(u_{h}, p_{h}\right) \in V_{h}^{n c} \times \mathcal{Q}_{h}$ to (5.1) satisfies, for all $\left(v_{h}, q_{h}\right) \in V_{h}^{n c} \times \mathcal{Q}_{h}$,

$$
\mu \int_{\Omega} D_{\mathcal{T}} u_{h}: D_{\mathcal{T}} v_{h} d x-\int_{\Omega} p_{h} \operatorname{div}_{\mathcal{T}} v_{h} d x+\int_{\Omega} \operatorname{div}_{\mathcal{T}} u_{h} q_{h} d x=\int_{\Omega} g v_{h} d x .
$$

Given the unique discrete solution $u_{h} \in H^{1}(\mathcal{T})^{n}$ and $p_{h} \in L_{0}^{2}(\Omega)$, set

$$
\sigma_{h}:=\mu D_{\mathcal{T}} u_{h}-p_{h} \mathrm{id} \in L
$$

and define the linear functional $\mathcal{R}_{e} s_{V}: V:=H_{0}^{1}(\Omega)^{n} \rightarrow \mathbb{R}$ by

$$
\mathcal{R}_{e} s_{V}(v)=\int_{\Omega}\left(g \cdot v-\sigma_{h}: D v\right) d x \quad \text { for } v \in V:=H_{0}^{1}(\Omega)^{n} .
$$

The theory of Section 3 shows that the norm of the residual $\mathcal{R} e s_{L}$ reads

$$
\left\|\mathcal{R} e s_{L}\right\|_{L^{*}} \approx\left\|D\left(\tilde{u}_{h}\right)-\operatorname{dev} D_{\mathcal{T}}\left(u_{h}\right)\right\|_{L^{2}(\Omega)} .
$$

Given any $\tilde{u}_{h} \in V$ with $\sigma:=\mu D u-p$ id, the unifying theory in the form of (1.7) and (5.4) prove

$\left\|\sigma-\sigma_{h}\right\|_{L}+\left\|u-\tilde{u}_{h}\right\|_{V} \lesssim\left\|D\left(\tilde{u}_{h}\right)-D_{\mathcal{T}}\left(u_{h}\right)\right\|_{L^{2}(\Omega)}+\left\|\operatorname{div}_{\mathcal{T}} u_{h}\right\|_{L^{2}(\Omega)}+\left\|\mathcal{R} e s_{V}(v)\right\|_{V^{*}}$.

5.3. Examples. This subsection lists some examples of nonconforming finite element schemes with (H1)-(H3) from the literature displayed in Table 5.1. Then, it follows from (5.9), the definitions of $\boldsymbol{\sigma}$ and $\boldsymbol{\sigma}_{h}$ with a straightforward investigation, Theorem 2.1, and Theorem 3.1, that

$$
\begin{aligned}
& \left\|D u-D_{\mathcal{T}} u_{h}\right\|_{L^{2}(\Omega)}+\left\|p-p_{h}\right\|_{L^{2}(\Omega)} \\
& \lesssim \min _{\tilde{u}_{h} \in V}\left\|D\left(\tilde{u}_{h}\right)-D_{\mathcal{T}}\left(u_{h}\right)\right\|_{L^{2}(\Omega)}+\left\|\operatorname{div}_{\mathcal{T}} u_{h}\right\|_{L^{2}(\Omega)}+\left\|\mathcal{R} e s_{V}(v)\right\|_{V^{*}} \\
& \lesssim \eta+\mu+\left\|\operatorname{div}_{\mathcal{T}} u_{h}\right\|_{L^{2}(\Omega)}+\operatorname{osc}(g) .
\end{aligned}
$$

This recovers the result from $[36,33]$ for the Crouzeix-Raviart element, and is new for five parallelogram elements of Subsubsection 5.3.2.

5.3.1. The Crouzeix-Raviart element. This is a triangular element with the velocity space

$$
V_{h}^{n c}:=V_{h}^{C R} \times V_{h}^{C R}
$$

for the space $V_{h}^{C R}$ from Subsection 4.3.1, and the piecewise constant pressure space $\mathcal{Q}_{h} \subset L_{0}^{2}(\Omega)$. Since $V_{h}^{c} \times V_{h}^{c} \subset V_{h}^{C R} \times V_{h}^{C R}$, there holds (H1)-(H3) with $\Pi=\mathrm{id}$.

5.3.2. Four parallelogram elements. There are four parallelogram elements in the literature including the parallelogram Han element, the parallelogram nonconforming rotated $(N R)$ element of Rannacher and Turek [60], the parallelogram $C J Y$ element [22], and the parallelogram constrained nonconforming rotated element of $\mathrm{Hu}$, Man and Shi [49]. These elements employ the piecewise constant pressure space 


\begin{tabular}{|c|c|c|c|}
\hline picture & name & reference & space \\
\hline & Crouzeix-Raviart & {$[35]$} & $V_{h}^{C R} \times V_{h}^{C R}$ \\
\hline & Han & {$[45]$} & $V_{h}^{\text {Han }} \times V_{h}^{\text {Han }}$ \\
\hline & NR & {$[60]$} & $V_{h}^{R T, A} \times V_{h}^{R T, A}$ \\
\hline & Hu-Man-Shi & {$[49]$} & $V_{h}^{C R T} \times V_{h}^{C R T}$ \\
\hline & CJY & {$[22]$} & $V_{h}^{D S S Y} \times V_{h}^{D S S Y}$ \\
\hline$\Delta .0$ & Kouhia-Stenberg & {$[54]$} & $V_{h}^{c} \times V_{h}^{C R}$ \\
\hline
\end{tabular}

TABle 5.1. Nonconforming Elements for the Stokes Problem (5.1) with (H1)-(H3) and the Error Estimate (5.10).

$\mathcal{Q}_{h} \subset L_{0}^{2}(\Omega)$. The velocity spaces for these methods are chosen from the following list.

$$
\begin{aligned}
V_{h}^{n c}:= & V_{h}^{\text {Han }} \times V_{h}^{\text {Han }}, V_{h}^{R T, A} \times V_{h}^{R T, A}, \\
& V_{h}^{C R T} \times V_{h}^{C R T}, V_{h}^{D S S Y} \times V_{h}^{D S S Y} .
\end{aligned}
$$

Herein $V_{h}^{\text {Han }}, V_{h}^{R T, A}, V_{h}^{C R T}$ and $V_{h}^{D S S Y}$ denote the nonconforming finite element spaces from the respective Subsubsections 4.3.3-4.3.6. Then there holds (H1)-(H3) with the canonical interpolation operators $\Pi$ for these nonconforming finite element spaces. The proof follows with the results of Section 4; further details are omitted.

Remark 5.1. The parallelogram nonconforming finite elements from [33] can also be analyzed in the present framework to recover the a posteriori error estimation on for the isotropic mesh therein.

5.4. The Kouhia-Stenberg element. The Stokes problem in its form (5.1) is equivalent to the symmetric form with $\varepsilon(u):=\operatorname{sym}(D u):=1 / 2\left(D u+D u^{T}\right)$ replacing $D u$ in (5.1). The velocity space [54] reads

$$
V_{h}^{n c}:=V_{h}^{c} \times V_{h}^{C R} \text {. }
$$

Since $V_{h}^{c} \times V_{h}^{c} \subset V_{h}^{c} \times V_{h}^{C R}$, there holds (H1)-(H3) with $\Pi=$ id, cf. [23].

\section{LiNEAR ELASTICITY}

This section is devoted to the Navier-Lamé equation and its locking-free nonconforming finite element approximation. The presented unifying theory leads to a posteriori error estimates which are robust with respect to the Lamé parameter $\lambda \rightarrow \infty$. Subsection 6.1 displays the model problem and Subsection 6.2 NCFEMs and their unifying error control. Subsection 6.3 presents some examples. Subsection 6.4 discusses the unsymmetric formulation for linear elasticity and the examples for this case are given in Subsection 6.5 
6.1. Model Problem. Adopt the notation of the previous sections and the following linear stress-strain relation, for $\lambda, \mu>0$,

$\mathbb{C} F:=\lambda \operatorname{tr}(F)$ id $+2 \mu F$ and $\mathbb{C}^{-1} F:=\frac{1}{2 \mu} F-\frac{\lambda}{2 \mu(n \lambda+2 \mu)} \operatorname{tr}(F)$ id, for $F \in \mathbb{R}^{n \times n}$.

The weak form of the linear elasticity problem reads: Given $g \in L^{2}(\Omega)^{n}$ find $u \in$ $V:=H_{0}^{1}(\Omega)^{n}$ with

$$
\int_{\Omega} \varepsilon(v): \sigma d x=\int_{\Omega} g \cdot v d x \text { and } \sigma=\mathbb{C} \varepsilon(u) \text { for all } v \in V .
$$

Define the operator $A: X=L \times V \rightarrow X^{*}$ for any $(\sigma, u) \in X$ by

$$
(A(\sigma, u))(\tau, v):=\left(\mathbb{C}^{-1} \sigma, \tau\right)_{L^{2}(\Omega)}-(\sigma, \varepsilon(v))_{L^{2}(\Omega)}-(\tau, \varepsilon(u))_{L^{2}(\Omega)} .
$$

Here, $L:=\left\{\sigma \in L^{2}\left(\Omega, \mathbb{R}_{\mathrm{sym}}^{n \times n}\right), \int_{\Omega} \operatorname{tr} \sigma d x=0\right\}$. The operator $A$ is linear, bounded, and bijective with $\lambda$-independent operator norms of $A$ and $A^{-1}[17,28]$.

6.2. Nonconforming finite element methods and unified a posteriori error estimators. With the nonconforming finite element approximation $u_{h} \in V_{h}^{n c}$ to $u$ and the discrete Green strain $\left.\varepsilon_{\mathcal{T}}(v):=\left(D_{\mathcal{T}} v+D_{\mathcal{T}} v^{T}\right)\right) / 2 \in L^{2}\left(\Omega ; \mathbb{R}_{\mathrm{sym}}^{n \times n}\right)$, set

$$
\sigma_{h}=2 \mu \varepsilon_{\mathcal{T}}\left(u_{h}\right)+\lambda \Pi_{2} \operatorname{div}_{\mathcal{T}} u_{h} \mathrm{id} .
$$

Throughout this section, $\Pi_{2}: L^{2}(\Omega) \rightarrow L^{2}(\Omega)$ denotes some reduction operators in the context of the locking phenomena, and the discrete stress $\sigma_{h}$ is supposed to satisfy

$$
\int_{\Omega} \sigma_{h}: \varepsilon_{\mathcal{T}}\left(v_{h}\right) d x=\int_{\Omega} g \cdot v_{h} d x \quad \text { for all } v_{h} \in V_{h}^{n c} .
$$

We define the continuous and discrete pressures as

$$
p=\lambda \operatorname{div} u \text { and } p_{h}=\lambda \Pi_{2} \operatorname{div}_{\mathcal{T}} u_{h} .
$$

Theorem 6.1. For any $\tilde{u}_{h} \in V$ there holds

$$
\begin{aligned}
& \left\|\varepsilon(u)-\varepsilon_{\mathcal{T}}\left(u_{h}\right)\right\|_{L^{2}(\Omega)}+\left\|p-p_{h}\right\|_{L^{2}(\Omega)}+\left\|\varepsilon\left(u-\tilde{u}_{h}\right)\right\|_{L^{2}(\Omega)} \\
& \approx\left\|\varepsilon_{\mathcal{T}}\left(u_{h}\right)-\varepsilon\left(\tilde{u}_{h}\right)\right\|_{L^{2}(\Omega)}+\left\|\mathcal{R} e s_{V}\right\|_{V^{*}}+\left\|\operatorname{div}_{\mathcal{T}} u_{h}-\Pi_{2} \operatorname{div}_{\mathcal{T}} u_{h}\right\|_{L^{2}(\Omega)} .
\end{aligned}
$$

Proof. The unifying theory with (1.7) and (6.3) reads in the present notations

$$
\left\|\sigma-\sigma_{h}\right\|_{L}+\left\|\varepsilon\left(u-\tilde{u}_{h}\right)\right\|_{L^{2}(\Omega)} \approx\left\|\mathbb{C}^{-1} \sigma_{h}-\varepsilon\left(\tilde{u}_{h}\right)\right\|_{L^{2}(\Omega)}+\left\|\mathcal{R} e s_{V}\right\|_{V^{*}} .
$$

Then the assertion follows from the definitions of $\sigma_{h}, \mathbb{C}^{-1}, p$, and $p_{h}$.

6.3. Examples. This subsection analyzes finite element methods depicted in Table 6.1 for the planar elasticity problem. These schemes satisfy (H1)-(H3). Then, the estimate (6.7) with Theorem 2.1 and Theorem 3.1 leads to

$$
\begin{aligned}
& \left\|\varepsilon(u)-\varepsilon_{\mathcal{T}}\left(u_{h}\right)\right\|_{L^{2}(\Omega)}+\left\|p-p_{h}\right\|_{L^{2}(\Omega)} \\
& \lesssim \min _{\tilde{u}_{h} \in V}\left\|\varepsilon_{\mathcal{T}}\left(u_{h}\right)-\varepsilon\left(\tilde{u}_{h}\right)\right\|_{L^{2}(\Omega)}+\left\|\mathcal{R}_{e} s_{V}\right\|_{V^{*}}+\left\|\operatorname{div}_{\mathcal{T}} u_{h}-\Pi_{2} \operatorname{div}_{\mathcal{T}} u_{h}\right\|_{L^{2}(\Omega)} \\
& \lesssim \mu+\eta+\left\|\operatorname{div}_{\mathcal{T}} u_{h}-\Pi_{2} \operatorname{div}_{\mathcal{T}} u_{h}\right\|_{L^{2}(\Omega)}+\operatorname{osc}(g) .
\end{aligned}
$$




\begin{tabular}{|c|c|c|c|}
\hline picture & name & reference & space \\
\hline & Kouhia-Stenberg & [54] & $V_{h}^{c} \times V_{h}^{C R}$ \\
\hline$:$ : : & Zhang & {$[75]$} & $V_{h}^{W i l} \times V_{h}^{W i l}$ \\
\hline Q. & Ming & {$[56]$} & $V_{h}^{c} \times V_{h}^{R T, A}$ \\
\hline
\end{tabular}

TABLE 6.1. Nonconforming Elements for the Linear Elasticity Problem (6.2) with (H1)-(H3) and the Error Estimate (6.9).

The error control for the Kouhia-Stenberg element has already been analyzed in [23]. The a posteriori error estimator (6.9) for the Falk elements, the Zhang element, and the Ming element is new.

6.3.1. The Falk elements. Two nonconforming triangular finite element methods are proposed in [41] for the linear elasticity equation for $k=2,3$ with $\Pi_{2}=\mathrm{id}$ and

$V_{h}^{n c}:=\left\{v \in L^{2}(\Omega)^{2}: \forall T \in \mathcal{T}_{h},\left.v\right|_{T} \in P_{k}(T)^{2}\right.$ and $v$ is continuous (res. vanishes) at the $k$ Gauss points on each interior (resp. boundary) edge $\}$.

Since $V_{h}^{c} \times V_{h}^{c} \subset V_{h}^{n c}$ there holds (H1)-(H3) with $\Pi=\mathrm{id}$.

6.3.2. The Kouhia-Stenberg element. This triangular element for the symmetric formulation (6.1) and $\Pi_{2}=\mathrm{id}[54]$ is defined by the nonconforming finite element space

$$
V_{h}^{n c}:=V_{h}^{c} \times V_{h}^{C R} .
$$

Since $V_{h}^{c} \times V_{h}^{c} \subset V_{h}^{c} \times V_{h}^{C R}$ there holds (H1)-(H3) with $\Pi=$ id, cf. also [23].

6.3.3. The Zhang element. This element is proposed in [75] based on the nonconforming quadrilateral Wilson element $[73,64]$ with $\Pi_{2}=\mathrm{id}$. In this element,

$$
V_{h}^{n c}:=V_{h}^{W i l} \times V_{h}^{W i l} .
$$

Since $V_{h}^{c} \times V_{h}^{c} \subset V_{h}^{W i l} \times V_{h}^{W i l}$ there holds (H1)-(H3) with $\Pi=$ id.

6.3.4. The Ming element. In Ming's dissertation [56], a parallelogram nonconforming element is proposed based on the nonconforming rotated $Q_{1}$ space from [60] for planar elasticity. The nonconforming finite element space reads

$$
V_{h}^{n c}:=V_{h}^{c} \times V_{h}^{R T, A}
$$

where $\Pi_{2}=\Pi_{0}: L^{2}(\Omega) \rightarrow Q_{0}$ denotes the piecewise constant projection operator with $Q_{0}$ the piecewise constant space. Following the arguments in Subsubection 4.3.4 and [31], one proves (H1)-(H3) for the associated interpolation operator $\Pi$. 


\begin{tabular}{|c|c|c|c|}
\hline picture & name & reference & space \\
\hline & Brenner-Sung & {$[19]$} & $V_{h}^{C R} \times V_{h}^{C R}$ \\
\hline & Lee-Lee-Sheen & {$[55]$} & $V_{h}^{R T, A} \times V_{h}^{R T, A}$ \\
\hline$\square$ & Hu-Man-Shi & {$[49]$} & $V_{h}^{C R T} \times V_{h}^{C R T}$ \\
\hline
\end{tabular}

TABLE 6.2. Nonconforming Elements for the Linear Elasticity Problem in Unsymmetric Formulation with (H1)-(H3) and the Error Estimate (6.19).

6.4. The unsymmetric formulation. For the pure Dirichlet boundary condition under consideration, one can use the equivalent unsymmetric formulation and then define the following formal stress-strain relation, for $F \in \mathbb{R}^{n \times n}$,

$$
\mathbb{C} F:=(\lambda+\mu) \operatorname{tr}(F) \text { id }+\mu F \text { and } \mathbb{C}^{-1} F:=\frac{1}{\mu} F-\frac{\lambda+\mu}{\mu(n \lambda+(n+1) \mu)} \operatorname{tr}(F) \text { id . }
$$

Given some nonconforming finite element space $V_{h}^{n c}$, the finite element solution $u_{h} \in V_{h}^{n c}$ satisfies

$$
\int_{\Omega} \sigma_{h}: D_{\mathcal{T}} v_{h} d x=\int_{\Omega} g \cdot v_{h} d x \quad \text { for all } v_{h} \in V_{h}^{n c} .
$$

Given the unique discrete solution $u_{h} \in V_{h}^{n c}$, set

$$
\sigma_{h}=\mu D_{\mathcal{T}} u_{h}+(\lambda+\mu) \Pi_{2} \operatorname{div}_{\mathcal{T}} u_{h} \mathrm{id},
$$

The continuous and discrete pressures reads

$$
p=(\lambda+\mu) \operatorname{div} u \text { and } p_{h}=(\lambda+\mu) \Pi_{2} \operatorname{div}_{\mathcal{T}} u_{h} .
$$

Define the operator $A: X=L \times V:=\left\{\tau \in L^{2}\left(\Omega, \mathbb{R}^{n \times n}\right), \int_{\Omega} \operatorname{tr} \tau d x=0\right\} \times H_{0}^{1}(\Omega)^{n} \rightarrow$ $X^{*}$ for any $(\sigma, u) \in X$ as

$$
(A(\sigma, u))(\tau, v):=\left(\mathbb{C}^{-1} \sigma, \tau\right)_{L^{2}(\Omega)}-(\sigma, D v)_{L^{2}(\Omega)}-(\tau, D u)_{L^{2}(\Omega)} .
$$

The arguments for the symmetric case in [17] show that the operator $A$ is linear, bounded, and bijective with $\lambda$-independent operator norms of $A$ and $A^{-1}$. Following the argument for the symmetric case, one proves

Theorem 6.2. For any $\tilde{u}_{h} \in V$ there holds that

$$
\begin{aligned}
& \left\|D u-D_{\mathcal{T}} u_{h}\right\|_{L^{2}(\Omega)}+\left\|p-p_{h}\right\|_{L^{2}(\Omega)}+\left\|D\left(u-\tilde{u}_{h}\right)\right\|_{L^{2}(\Omega)} \\
& \quad \approx\left\|D_{\mathcal{T}} u_{h}-D \tilde{u}_{h}\right\|_{L^{2}(\Omega)}+\left\|\mathcal{R} e s_{V}\right\|_{V^{*}}+\left\|\operatorname{div}_{\mathcal{T}} u_{h}-\Pi_{2} \operatorname{div}_{\mathcal{T}} u_{h}\right\|_{L^{2}(\Omega)} .
\end{aligned}
$$

6.5. Examples. Three nonconforming finite elements are listed below as examples with the unsymmetric formulation and are summarized in Table 6.2. There holds that

$$
\begin{aligned}
& \left\|D u-D_{\mathcal{T}} u_{h}\right\|_{L^{2}(\Omega)}+\left\|p-p_{h}\right\|_{L^{2}(\Omega)} \\
& \lesssim \mu+\eta+\left\|\operatorname{div}_{\mathcal{T}} u_{h}-\Pi_{2} \operatorname{div}_{\mathcal{T}} u_{h}\right\|_{L^{2}(\Omega)}+\operatorname{osc}(g) .
\end{aligned}
$$

This a posteriori error estimator is brand new for these elements. 
6.5.1. The Brenner-Sung element. This triangular element is proposed in [19] with $\Pi_{2}=\mathrm{id}$, and

$$
V_{h}^{n c}:=V_{h}^{C R} \times V_{h}^{C R} .
$$

Since $V_{h}^{c} \times V_{h}^{c} \subset V_{h}^{C R} \times V_{h}^{C R}$ there holds (H1)-(H3) with $\Pi=\mathrm{id}$.

6.5.2. The Lee-Lee-Sheen element. In this parallelogram element [55], both components of the displacement are approximated by the nonconforming rotated $Q_{1}$ space from [60], namely

$$
V_{h}^{n c}:=V_{h}^{R T, A} \times V_{h}^{R T, A} .
$$

The reduction integration operator is the same as in the Ming elements. (H1)-(H3) is satisfied by this element with the canonical interpolation operator $\Pi$ for $V_{h}^{n c}$. It follows the arguments for the nonconforming rotated $Q_{1}$ element in Subsubsection 4.3.4.

6.5.3. The Hu-Man-Shi element. This parallelogram element is designed in [49] without reduction integration. The nonconforming finite element space is the constrained nonconforming rotated $Q_{1}$ from [50]. There also holds (H1)-(H3) with the canonical interpolation operator $\Pi$. The proof can be found in Subsubsection 5.3.2.

Remark 6.1. Our conditions and therefore analysis in this paper can be extended to other nonstandard finite element methods for the elasticity, for instance, the Wang-Qi element from [70] and the enhanced strain finite element from [63, 17].

\section{ACKNOWLEDGMENTS}

The second author JH thankfully acknowledges the Alexander von Humboldt Fellowship during his stay at the Department of Mathematics of Humboldt-Universität zu Berlin, Germany.

\section{REFERENCES}

[1] M. Ainsworth and J.T. Oden, A posteriori error estimation in finite element analysis, WileyInterscience [John Wiley \& Sons], New York, 2000.

[2] M. Ainsworth, Robust a posteriori error estimation for nonconforming finite element approximation, Preprint available at http://www.maths.strath.ac.uk/ aas98107/papers.html.

[3] M. Ainsworth, A posteriori error estimation for non-conforming quadrilateral finite elements, Int. J. of Numerical Analysis and Modeling, 2 (2005), 1-18.

[4] Douglas N. Arnold, An interior penalty finite element method with discontinuous elements, IAM J. Numer. Anal. 19 (1982), 742-760.

[5] Douglas N. Arnold, Franco Brezzi, Bernardo Cockburn, and Donatella Marini, Discontinuous Galerkin methods for elliptic problems, Discontinuous Galerkin Methods: Theory, Computation and Applications, B. Cockburn, G. Karniadakis, and C. W. Shu, eds., ecture Notes in Computational Science and Engineering 11, Springer-Verlag, New York, 2000, 89-101.

[6] I. Babuška and T.Strouboulis, The Finite Element Method and its Reliability, The Clarendon Press Oxford University Press, 2001.

[7] G.Baker, Finite element methods for elliptic equations using nonconforming elements, Math.Comp.,31(1977), 45-59.

[8] G.A. Baker, W.N. Jureidini, and O.A. Karakashian, Piecewise solenoidal vector fields and the Stokes problem, SIAM J. Numer. Anal. 27 (1990), 1466-1485. 
[9] R. Becker, P. Hansbo, and M. Larson, Energy norm a posteriori error estimation for. discontinuous Galerkin methods, Comput. Methods Appl. Mech. Engrg., 192(2003), 723-733.

[10] R. Becker and R. Rannacher, An optimal control approach to a posteriori error estimation in finite element methods, Acta Numerica, Cambridge University Press, 2001, 1-102.

[11] C. Bernardi, V. Girault, A local regularisation operator for triangular and quadrilateral finite elements, SIAM J. Numer. Anal., 35 (1998), 1893-1916.

[12] C.Bernardi and F.Hecht, Error indicators for the mortar finite element discretization of the Laplace equation, Math. Comp. 71(2002), 1371-1403.

[13] C. Bernardi, Y. Maday, and A. T. Patera. Domain decomposition by the mortar element method. In H. Kaper et al., editor, Asymptotic and numerical methods for partial differential equations and their applications, pages 269-286, Dordrecht, 1993. Reidel.

[14] C. Bernardi, Y. Maday, and A. T. Patera. A new nonconforming approach to domain decomposition: the mortar element method. In Nonlinear partial differential equations and their applications, pages 13-51, Paris, 1994.

[15] C. Bernardi, R.G. Owens, J. Valenciano - An error indicator for mortar element solutions to the Stokes problem, Internal Report 99030, Laboratoire d'Analyse Numrique, Université Pierre et Marie Curie, Paris (1999).

[16] D. Braess, Finite Elements, Cambridge University Press, 1997.

[17] D. Braess, C. Carstensen, B.D. Reddy, Uniform convergence and a posteriori error estimators for the enhanced strain finite element method, Numer.Math., 96(2004), 461-479.

[18] S.C. Brenner, L.R. Scott, The Mathematical Theory of Finite Element Methods, Springer Verlag, 2nd Edition, 2002.

[19] S.C. Brenner, L.Y. Sung, Linear finite element methods for planar linear elasticity, Math.Comp., 59(1992), 321-338.

[20] F. Brezzi, M. Fortin, Mixed and Hybrid Finite Element Methods, Springer, Berlin, 1991.

[21] Rommel Bustinza, Gabriel N. Gatica and Bernardo Cockburn, An a posteriori error estimate for the local discontinuous Galerkin method applied to linear and nonlinear diffusion problems, J.Scientific Computing, 22(2005),147-185

[22] Z. Cai, J. Douglas, Jr., X. Ye, A stable nonconforming quadrilateral finite element method for the stationary Stokes and Navier-Stokes equations, CaLcoLo, 36(1999), 215-232.

[23] C. Carstensen, A unifying theory of a posteriori finite element error control, Numer.Math, $100(2005), 617-637$.

[24] C. Carstensen, Quasi-interpolation and a posteriori error analysis in finite element methods, M2AN Math. Model. Numer. Anal., 33 (1999), 1187-1202.

[25] C. Carstensen, S. Bartels, Each averaging technique yields reliable a posteriori error control in FEM on unstructured grids. Part I: Low order conforming, nonconforming, and mixed FEM, Math. Comp., 71 (2002), 945-969.

[26] C. Carstensen, S. Bartels and G. Dolzmann, A posteriori error estimates for nonconforming finite element methods, Numer. Math., 92 (2002), 233-256

[27] C. Carstensen, S. Bartels, S. Jansche, A posteriori error estimates for nonconforming finite element methods, Numer. Math., 92 (2002), 233-256.

[28] C. Carstensen, G. Dolzmann, A posteriori error estimates for mixed FEM in elasticity, Numer.Math., 81(1998), 187-209.

[29] C. Carstensen and S.A. Funken, A posteriori error control in low-order finite element discretisations of incompressible stationary flow problems, Math. Comp., 70(2001), 1353-1381.

[30] C. Carstensen and S.A. Funken. Averaging technique for FE-a posteriori error control in elasticity. Part I: Conforming FEM. Comput. Methods Appl. Mech. Engrg., 190(2001), 24832498. Part II: $\lambda$-independent estimates, Comput. Methods Appl. Mech. Engrg., 190(2001), 4663-4675. Part III: Locking-free conforming FEM. Comput. Methods Appl. Mech. Engrg., 191(2001), 861-877.

[31] C. Carstensen, Jun Hu, A. Orlando, Framework for the a posteriori error analysis of nonconforming finite elements, Preprint (2005-11), Department of Mathematics, Humboldt University of Berlin (2005), accepted for publication by SIAM J.Numer.Anal., 2006. 
[32] C. Carstensen and R. Verfürth, R, Edge residuals dominate a posteriori error estimates for low order finite element methods, SIAM J. Numer. Anal., 36(1999), 1571-1587.

[33] E.Creusé, G.Kunert and S.Nicaise, A posteriori error estimation for the Stokes problem: Anisotropic and isotropic discretications, M3AS, 14(2004), 1-48.

[34] M. Crouzeix, R.S. Falk, Nonconforming finite elements for the Stokes problem, Math.Comp., $52(1989), 437-456$.

[35] M. Crouzeix, P.-A. Raviart, Conforming and nonconforming finite element methods for solving the stationary Stokes equations, RAIRO Anal. Numér., 7 (1973), 33-76.

[36] E. Dari, R. Duran, C. Padra, Error estimators for nonconforming finite element approximations of the Stokes problem, Math. Comp., 64 (1995), 1017-1033.

[37] E. Dari, R. Duran, C. Padra, V. Vampa, A posteriori error estimators for nonconforming finite element methods, Math. Model. Numer. Anal., 30 (1996), 385-400.

[38] J. Douglas, Jr. and T. Dupont, Interior penalty procedures for elliptic and parabolic Galerkin methods, In Lectures Notes in Physics, Vol. 58. Springer-Verlag, 1976.

[39] R.H.W. Hoppe and B. Wohlmuth, Element-orientated and edge-orientated local error estimates for nonconforming finite element methods, Math. Modeling Numer. Anal., 30(1996), $237-263$.

[40] J. Douglas Jr, J.E. Santos, D. Sheen, X. Ye, Nonconforming Galerkin methods based on quadrilateral elements for second order elliptic problems, Math. Model. Numer. Anal., 33 (1999), 747-770.

[41] R.S. Falk, Nonconforming finite element methods for the equations of linear elasticity, Math. Comp., 57(1991), 529-550.

[42] M. Grajewski, J.Hron and Stefan Turek, Numerical analysis for a new non-conforming linear finite element on quadrilaterals, Journal of Computational and Applied Mathematics, In Press.

[43] M. Grajewski, J.Hron and Stefan Turek, Dual Weighted a posteriori error estimation for a new nonconforming linear finite element on quadrilaterals, www.mathematik.unidortmund.de/lsiii/static/ showpdffile_GrajewskiHronTurek2004.pdf

[44] V. Girault, P.-A. Raviart, Finite Element Methods for Navier-Stokes Equations, Springer Verlag, 1986.

[45] Hou-De Han, Nonconforming elements in the mixed finite element method, J. Comp. Math., 2 (1984), 223-233

[46] Paul Houston, Dominik Schotzau an Thomas P. Wihler, Energy norm shape a posteriori error estimation for mixed discontinuous Galerkin approximations of the Stokes problem, J.Scientific Computing, 22(2005), 347-370 .

[47] Paul Houston, Dominik Schotzau and Thomas P. Wihler, An hp-adaptive mixed discontinuous Galerkin FEM for nearly incompressible linear elasticity, Comp.Methods Appl.Mech.Engrg., 195(2006), 224-3246.

[48] Paul Houston, Dominik Schotzau and Thomas P. Wihler, Energy norm a posteriori error estimation of hp-adaptive discontinuous Galerkin methods for elliptic problems, to appear in M3AS.

[49] Jun Hu, Hong-Ying Man and Zhong-Ci Shi, Constrained nonconforming rotated $Q_{1}$ element for Stokes flow and planar elasticity, Mathematica Numerica Sinica (in Chinese), 27(2005), 311-324.

[50] Jun Hu, Zhong-Ci Shi, Constrained quadrilateral nonconforming rotated $Q_{1}$-element, J. Comp. Math., 23(2005), 561-586.

[51] G. Kanschat, F.-T. Suttmeier, A posteriori error estimates for nonconforming finite element schemes, Calcolo, 36 (1999), 129-141.

[52] O.A. Karakashian and W.N. Jureidini, A nonconforming finite element method for the stationary Navier-Stokes equations, SIAM J. Numer. Anal. 35 (1998), 93-120.

[53] O.A. Karakashian and F. Pascal, A posteriori error estimates for a discontinuous Galerkin approximation of second-order elliptic problems. SIAM J. Numer. Anal., 41(2003), 23742399. 
[54] R. Kouhia, R. Stenberg, A linear nonconforming finite element method for nearly incompressible elasticity and Stokes flow, Comput.Methods Appl.Mech.Engrg., 124(1995), 195-212.

[55] C.O. Lee, J. Lee, D.W. Sheen, A locking-free nonconforming finite element method for planar linear elasticity, Advances in Computational Mathematics, 19(2003), 277-291.

[56] Ping-Bing Ming, Nonconforming finite element vs locking problem, Doctorate Dissertation (in Chinese), Institute of Computational Mathematics, Chinese Academy of Science (1999).

[57] Q.Lin, L.Tobiska and A.Zhou, On the superconvergence of nonconforming low order finite elements applied to the Poisson equation, IMA.J.Numer.Anal.,25(2005), pp. 160-181.

[58] S.Nicaise and E.Creusé, Isotropic and anisotropic a posteriori error estimation for the mixed finite element method of second order operators in divergence form, ETNA, 23(2006), 38-62.

[59] C. Park, D. Sheen, P1-nonconforming quadrilateral finite element methods for second-order elliptic problems, SIAM J. Numer. Anal., 41 (2003), 624-640.

[60] R. Rannacher, S. Turek, Simple nonconforming quadrilateral Stokes element, Numer. Meth. PDE, 8 (1992), 97-111.

[61] B. Riviere and M.F. Wheeler, A posteriori error estimates for a discontinuous Galerkin method applied to elliptic problems. Comput. Math. Appl., 46(2003),141-163.

[62] B. Riviere and M.F. Wheeler, A posteriori error estimates and mesh adaptation strategy for discontinuous Galerkin methods applied to diffusion problems, Computers \& Mathematics with Applications, 46(2003), 141-163.

[63] J.C. Simo, M.S.Rifai, A class of mixed assumed strain methods and the method of incompatible modes, Inter.J.Numer.Methods Engrg., 29(1990), 1595-1638.

[64] Zhong-Ci Shi, A convergence condition for quadrilateral Wilson element, Numer.Math., 44(1984), 349-361.

[65] Zhong-Ci Shi, A remark on the optimal order of convergence of Wilson nonconforming element, Mathematica Numerica Sinica (in Chinese), 8(1986), 159-163.

[66] Zhong-Ci Shi, The F-E-M-Test for nonconforming finite elements, Math. Comp., 49(1987), 391-405.

[67] R. Stenberg, A family of mixed finite elements for the elasticity problem, Numer.Math., 53(1988), 513-538.

[68] R. Verfürth, A Review of A Posteriori Error Estimation and Adaptive Mesh-Refinement Techniques, Wiley-Teubner, 1996.

[69] R. Verfürth, (1989). A posteriori error estimators for the Stokes equations, Numer. Math., $55(1989), 309-325$.

[70] Lie-Heng Wang, He Qi, On locking-free finite element schemes for the pure displacement boundary value problem in the planar elasticity, Mathematica Numerica Sinica (in Chinese), 24(2002), 243-256.

[71] M.F. Wheeler, An elliptic collocation-finite element method with interior penalties, SIAM J.Numer.Anal, 15 (1978), 152-161.

[72] Thomas P. Wihler, Locking-free adaptive discontinuous Galerkin FEM for linear elasticity problems, Math.Comp.,75(2006), 1087-1102.

[73] E.L. Wilson, R.L. Taylor, W. Doherty, J. Ghaboussi, Incompatible displacement models. In S.J. Fenves, N. Perrone, A.R. Robinson, W.C. Schnobrich, editors, Numerical and Computer Methods in Structural Mechanics, 43-57, Academic Press, 1973.

[74] B. Wohlmuth - A residual based error estimator for mortar finite element discretizations, Numer. Math. 84 (1999), 143-171.

[75] Zhi-Min Zhang, Analysis of some quadrilateral nonconforming elements for incompressible elasticity, SIAM.J.Numer.Anal., 34(1997), 640-663.

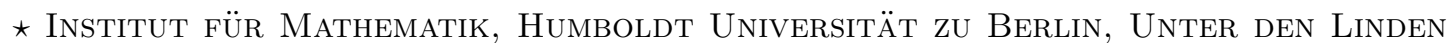

6, D-10099 Berlin, Germany

E-mail address: cc@mathematik.hu-berlin.de 
$\ddagger$ LMam and School of Mathematical Sciences, Peking University, Beijing 100871, People's Rebublic of China

E-mail address: hujun@math.pku.edu.cn 